\title{
PESSTO: survey description and products from the first data release by the Public ESO Spectroscopic Survey of Transient Objects $^{\star, \star \star}$
}

\author{
S. J. Smartt ${ }^{1}$, S. Valenti ${ }^{2,3}$, M. Fraser ${ }^{4}$, C. Inserra ${ }^{1}$, D. R. Young ${ }^{1}$, M. Sullivan ${ }^{5}$, A. Pastorello ${ }^{6}$, S. Benetti $^{6}$, \\ A. Gal-Yam ${ }^{7}$, C. Knapic ${ }^{8}$, M. Molinaro ${ }^{8}$, R. Smareglia ${ }^{8}$, K. W. Smith ${ }^{1}$, S. Taubenberger ${ }^{9}$, O. Yaron ${ }^{7}$, J. P. Anderson ${ }^{10}$, \\ C. Ashall ${ }^{18}$, C. Balland ${ }^{11}$, C. Baltay ${ }^{12}$, C. Barbarino ${ }^{13,14}$, F. E. Bauer ${ }^{15,16,17,}$, S. Baumont ${ }^{11}$, D. Bersier ${ }^{18}$, \\ N. Blagorodnova ${ }^{4}$, S. Bongard ${ }^{11}$, M. T. Botticella ${ }^{13}$, F. Bufano ${ }^{19}$, M. Bulla ${ }^{1}$, E. Cappellaro ${ }^{6}$, H. Campbell ${ }^{4}$, \\ F. Cellier-Holzem ${ }^{11}$, T.-W. Chen ${ }^{1}$, M. J. Childress ${ }^{20,32}$, A. Clocchiatti ${ }^{15,16}$, C. Contreras ${ }^{43,44}$, M. Dall'Ora ${ }^{13}$, \\ J. Danziger ${ }^{8}$, T. de Jaeger ${ }^{23,37}$, A. De $\mathrm{Cia}^{7}$, M. Della Valle ${ }^{13}$, M. Dennefeld ${ }^{21}$, N. Elias-Rosa ${ }^{6,22}$, N. Elman ${ }^{12}$, \\ U. Feindt ${ }^{39,40}$, M. Fleury ${ }^{11}$, E. Gall ${ }^{1}$, S. Gonzalez-Gaitan ${ }^{23,37}$, L. Galbany ${ }^{23,37}$, A. Morales Garoffolo ${ }^{22}$, L. Greggio ${ }^{6}$, \\ L. L. Guillou ${ }^{11}$, S. Hachinger ${ }^{33,34,6}$, E. Hadjiyska ${ }^{12}$, P. E. Hage ${ }^{11}$, W. Hillebrandt ${ }^{9}$, S. Hodgkin ${ }^{4}$, E. Y. Hsiao ${ }^{44,43}$, \\ P. A. James ${ }^{18}$, A. Jerkstrand ${ }^{1}$, T. Kangas ${ }^{36}$, E. Kankare ${ }^{1}$, R. Kotak ${ }^{1}$, M. Kromer ${ }^{26}$, H. Kuncarayakti ${ }^{23,37}$, \\ G. Leloudas ${ }^{25,7}$, P. Lundqvist ${ }^{26}$, J. D. Lyman ${ }^{45}$, I. M. Hook ${ }^{27,28}$, K. Maguire ${ }^{29}$, I. Manulis ${ }^{7}$, S. J. Margheim ${ }^{30}$, \\ S. Mattila ${ }^{24}$, J. R. Maund ${ }^{1}$, P. A. Mazzali ${ }^{18}$, M. McCrum ${ }^{1}$, R. McKinnon ${ }^{12}$, M. E. Moreno-Raya ${ }^{42}$, M. Nicholl ${ }^{1}$, \\ P. Nugent ${ }^{31,41}$, R. Pain ${ }^{11}$, G. Pignata ${ }^{19,16}$, M. M. Phillips ${ }^{43}$, J. Polshaw ${ }^{1}$, M. L. Pumo ${ }^{6}$, D. Rabinowitz ${ }^{12}$, E. Reilly ${ }^{1}$, \\ C. Romero-Cañizales ${ }^{15,16}$, R. Scalzo ${ }^{20}$, B. Schmidt ${ }^{20}$, S. Schulze ${ }^{15,16}$, S. Sim ${ }^{1}$, J. Sollerman ${ }^{26}$, F. Taddia ${ }^{26}$, \\ L. Tartaglia ${ }^{6,38}$, G. Terreran ${ }^{1,6}$, L. Tomasella ${ }^{6}$, M. Turatto ${ }^{6}$, E. Walker ${ }^{12}$, N. A. Walton ${ }^{4}$, L. Wyrzykowski ${ }^{35,4}$, \\ F. Yuan ${ }^{20,32}$, and L. Zampieri ${ }^{6}$ \\ (Affiliations can be found after the references)
}

Received 29 October 2014 / Accepted 17 April 2015

\begin{abstract}
Context. The Public European Southern Observatory Spectroscopic Survey of Transient Objects (PESSTO) began as a public spectroscopic survey in April 2012. PESSTO classifies transients from publicly available sources and wide-field surveys, and selects science targets for detailed spectroscopic and photometric follow-up. PESSTO runs for nine months of the year, January - April and August - December inclusive, and typically has allocations of 10 nights per month.

Aims. We describe the data reduction strategy and data products that are publicly available through the ESO archive as the Spectroscopic Survey data release 1 (SSDR1).

Methods. PESSTO uses the New Technology Telescope with the instruments EFOSC2 and SOFI to provide optical and NIR spectroscopy and imaging. We target supernovae and optical transients brighter than $20.5^{\mathrm{m}}$ for classification. Science targets are selected for follow-up based on the PESSTO science goal of extending knowledge of the extremes of the supernova population. We use standard EFOSC2 set-ups providing spectra

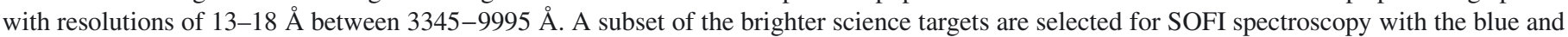
red grisms (0.935-2.53 $\mu$ m and resolutions $23-33 \AA$ ) and imaging with broadband $J H K_{\mathrm{s}}$ filters.

Results. This first data release (SSDR1) contains flux calibrated spectra from the first year (April 2012-2013). A total of 221 confirmed supernovae were classified, and we released calibrated optical spectra and classifications publicly within $24 \mathrm{~h}$ of the data being taken (via WISeREP). The data in SSDR1 replace those released spectra. They have more reliable and quantifiable flux calibrations, correction for telluric absorption, and are made available in standard ESO Phase 3 formats. We estimate the absolute accuracy of the flux calibrations for EFOSC2 across the whole survey in SSDR1 to be typically $\sim 15 \%$, although a number of spectra will have less reliable absolute flux calibration because of weather and slit losses. Acquisition images for each spectrum are available which, in principle, can allow the user to refine the absolute flux calibration. The standard NIR reduction process does not produce high accuracy absolute spectrophotometry but synthetic photometry with accompanying $J H K_{\mathrm{s}}$ imaging can improve this. Whenever possible, reduced SOFI images are provided to allow this.

Conclusions. Future data releases will focus on improving the automated flux calibration of the data products. The rapid turnaround between discovery and classification and access to reliable pipeline processed data products has allowed early science papers in the first few months of the survey.
\end{abstract}

Key words. instrumentation: spectrographs - methods: data analysis - techniques: spectroscopic - surveys - supernovae: general

^ Based on observations collected at the European Organisation for Astronomical Research in the Southern Hemisphere, Chile, as part of programme 188.D-3003 (PESSTO).

$\star \star$ wWw. pessto.org

\section{Introduction}

The search for transient phenomena in the Universe has entered a new era, with the construction and operation of dedicated wide-field optical telescopes, coupled with large format digital 
cameras and rapid data analysis pipelines. The current surveys in operation with $0.7-1.8 \mathrm{~m}$ aperture wide-field telescopes combined with cameras covering 5-10 square degrees that are actively searching for transients are the Palomar Transient Factory (PTF; Law et al. 2009), the Pan-STARRS1 survey (PS1; Kaiser et al. 2010), the Catalina Real Time Survey (CRTS; Drake et al. 2009), the La Silla QUEST survey (LSQ; Baltay et al. 2013), and the SkyMapper survey (Keller et al. 2007). With exposure times of 30-60 s, these surveys can reach magnitudes between 19-21, and can each cover around 1000-6000 square degrees per night depending on the telescope. Each survey runs transient object detection pipelines, with differing methodology, but a common factor amongst the surveys is the requirement for rapid spectroscopic observations of new transients. The simplest initial characteristic of a transient that is immediately required is distance, to provide an estimate of emitted energy, albeit initially in the narrow wavelength region of the optical domain.

The PTF built dedicated fast follow-up into the factory element of the project from the outset, successfully combining transient detection on the Palomar $1.2 \mathrm{~m}$ Schmidt with spectroscopic follow-up on accessible 2-4 m telescopes within their consortium. (e.g. Rau et al. 2009; Gal-Yam et al. 2011; Nugent et al. 2011). PS1 has concentrated efforts on higher redshift regimes, covering similar volume to PTF's imaging survey with its 10 Medium Deep fields, daily cadence, and follow-up on 4m, 6m, and $8 \mathrm{~m}$ telescopes (e.g. Botticella et al. 2010; Chomiuk et al. 2011; Berger et al. 2012; Gezari et al. 2012). Other projects are using smaller aperture $(\sim 0.1-0.4 \mathrm{~m})$ telescopes or cameras to cover wider fields to shallower depths. The MASTER project employs a number of $40 \mathrm{~cm}$ telescopes and $7 \mathrm{~cm}$ cameras in the northern hemisphere, finding optical transients down to $\sim 20 \mathrm{mag}$ (Lipunov et al. 2010). The ASAS-SN project is now running two $14 \mathrm{~cm}$ telescopes in the northern and southern hemispheres, successfully finding transients brighter than $V \sim 17$ (Shappee et al. 2014) with the aim of being all sky to approximately this flux limit. The very successful OGLE project is now producing extragalactic transients in its $\sim 700$ square degree footprint of the OGLE-IV survey with a $1.3 \mathrm{~m}$ telescope and 1.4 square degree field of view (Kozłowski et al. 2013; Wyrzykowski et al. 2014).

Between them, these synoptic surveys are discovering new classes of transients that challenge our ideas of the physics of stellar explosions. The long running and very successful nearby supernova (SN) searches of LOSS (Li et al. 2011; Leaman et al. 2011) and CHASE (Pignata et al. 2009), are aided by the large community of well equipped and experienced amateur astronomers throughout the world who have also increased their detection limits to provide some critical scientific data (see e.g. Itagaki's contribution to Pastorello et al. 2007). These have targeted bright galaxies (within about $\sim 100 \mathrm{Mpc}, z \lesssim 0.025$ ) for the obvious reasons that they host much of the mass and star formation in the local Universe. The SN population in these galaxies are well studied (Li et al. 2011), and the progenitor stars of many core-collapse SNe have been discovered (Smartt 2009) leading to physical insights into the explosions and the progenitor population. However, surprises still appear in these galaxy focused surveys such as the faint hydrogen poor SNe (Valenti et al. 2009; Kasliwal et al. 2010). The origins of some of these are disputed and it is not clear if they are thermonuclear explosions of white dwarfs or related to core-collapse. The nature of faint transients such as that in M85, which are between 1-2 mag brighter than classical novae, have been suggested as potential stellar mergers rather than SNe (Kulkarni et al. 2007).

The new wide-field transient searches discover transients with no galaxy bias and fainter limiting magnitudes, and probe shorter timescales. This has opened up a new window on the transient Universe - and the physical diversity discovered thus far is challenging the paradigms we hold for stellar deaths. It is likely that we are witnessing the diversity in the transient Universe that depends on stellar mass, metallicity, binarity, mass-loss rates, and rotation rates of the progenitor systems. However the biggest challenge in the field is now linking the discoveries to rapid spectroscopic and multi-wavelength follow-up. PTF has discovered and spectroscopically classified 2288 transients over the years 2010-2012, which made up 25-50\% of all SNe found and classified in this period (Sullivan 2013). PS1 has discovered over 4000 transients, with spectroscopy of $10 \%$. Still many transients go unclassified and wide-field searches in the south are only just beginning. Furthermore, we will soon enter the era of multi-messenger astronomy, which aims to link electromagnetic detections to gravitational wave, neutrino, and high-energy cosmic ray sources (O'Brien \& Smartt 2013).

In response to the first call by the European Southern Observatory (ESO) for public spectroscopic surveys, and particularly prompted by the opportunities provided by the LSQ and SkyMapper surveys, we proposed PESSTO (the Public ESO Spectroscopic Survey of Transient Objects). This built on the work and broad european consortium gathered together in the ESO Large Programme "Supernova Variety and Nucleosynthesis Yields" lead by Benetti (ESO 184.D-1140, 30 nights/yr allocated at ESO-NTT; e.g. see Pastorello et al. 2013). PESSTO was accepted by ESO as one of two public surveys, the other being the Gaia-ESO survey using FLAMES on the Very Large Telescope. PESSTO was awarded 90 nights per year on the New Technology Telescope (NTT) initially for two years, which has been renewed to four years (2012-2016). The science goal of PESSTO is to provide a public spectroscopic survey to deliver detailed, high-quality, time series optical+NIR spectroscopy of about 150 optical transients covering the full range of parameter space that the surveys now deliver : luminosity, host metallicity, explosion mechanisms. The PESSTO team is composed of the major supernovae research teams in the ESO community and rapid access to the reduced data is an integral part of the project. To date, ten papers based primarily on PESSTO data have been accepted in refereed journals (Fraser et al. 2013; Maund et al. 2013; Childress et al. 2013; Valenti et al. 2014a,b; Benetti et al. 2014; Inserra et al. 2014; Maguire et al. 2013; Scalzo et al. 2014; Nicholl et al. 2014)

\section{Description of the survey and data reduction pipeline}

PESSTO is allocated 90 nights per year, in visitor mode, on the ESO NTT. There are no observations planned during the months of May, June, and July because the Galactic centre is at optimal right ascension. These three months make it more difficult to search for extragalactic $\mathrm{SNe}$ and there is large time pressure from the ESO community for Milky Way stellar science. PESSTO is typically allocated 10 nights per month split into three sub-runs of $4 \mathrm{~N}, 3 \mathrm{~N}$ and $3 \mathrm{~N}$. The middle sub-run is usually dark time, while the two others are grey/bright with the moon up for around $50 \%$ of the time. The instruments used are EFOSC2 and SOFI and both spectroscopy and imaging modes are employed. The PESSTO collaboration host public webpages which includes information on night reports, observing conditions, observing with the NTT and instructions for downloading the data reduction pipeline. This information is udpated during 
the survey and users should read this document with the information on www . pessto . org and the wiki pages that the homepage points to.

\subsection{Target selection and strategy}

We have built a web-based data aggregator that works to pool various institutional and transient survey websites, alongside astronomical transient alert resources such as The Astronomers Telegram $(\mathrm{ATel})^{1}$ and the IAU Central Bureau for Electronic Telegrams $(\mathrm{CBET})^{2}$ services. This aggregator (the PESSTO Marshall), cross-correlates all transient event metadata coming from these various sources, grouping duplicate objects together, and presenting the user with a detailed overview of what is currently known about each transient event.

The PESSTO Marshall also provides a structured workflow which allows users to both promote objects they wish to be classified with the NTT and to track observations of objects they have chosen for detailed follow-up. The Marshall also works well as a collaboration and communication platform for the PESSTO community, allowing users to comment on objects they are interested in, to append useful object metadata, to state their intentions about individual objects and to provide observers at the NTT with detailed instructions as to what observations they require.

The major science goal of PESSTO is to extend the detailed time series data for unusual and rare transient events that challenge our understanding of the two standard physical mechanisms for $\mathrm{SNe}$ : core-collapse and thermonuclear. Furthermore, nearby supernovae allow for more detailed study such as progenitor detections and multi-wavelength (x-ray to radio) follow-up that probes both the explosion mechanisms and shock physics. To this end, the PESSTO collaboration has formed Science groups who study the classification spectra and promote targets to "PESSTO Key Science Targets" on the basis of them falling into these areas. PESSTO takes input sources for classification from all publicly available sources, and has partnerships in particular with the LSQ, SkyMapper and OGLE-IV surveys for access to its targets as early as possible. The public feed of CRTS targets has also proved to be a very valuable source of targets. PESSTO employs a magnitude limit for classification of 20.5 in $B V R$ or unfiltered CCD magnitudes and particularly looks to prioritise targets according to the following criteria

- Distance $(d<40 \mathrm{Mpc})$ and very high priority for candidates with $d<25 \mathrm{Mpc}$.

- Young phase: non-detection at $<7$ days, or fast rise time ( $>0.5 \mathrm{mag} / \mathrm{day})$ or sharp drops in magnitude over short timescales.

- Luminosity extremes: objects with expected $M<-19.5$ and $M>-15.5$. This is difficult to implement as the distance or redshift of the host is required, but when possible it is used.

- Fast declining lightcurves $(\Delta \mathrm{mag}>1 \mathrm{mag} / 5$ days) or very slow-rising lightcurves $\left(t_{\text {rise }}>30\right.$ days).

- Variability history - for example pre-discovery outburst such as SN 2006jc, SN 2009ip, SN 2010mc (Pastorello et al. 2007, 2013; Fraser et al. 2013; Ofek et al. 2013).

- XRF and GRB alerts: these have not yet been observed, because of a lack of targets at the right magnitude and availability.

\footnotetext{
1 http://www. astronomerstelegram.org

2 http://www. cbat.eps.harvard.edu/cbet/RecentCBETs. html
}

- Peculiar host environments: for example low-luminosity galaxies: $M_{B}>-18$ or hostless transients; remote locations in E/S0 galaxies and in the halos of spirals $(d>20 \mathrm{kpc})$ from the nucleus; enhanced star formation environments such as Arp galaxies; interacting systems or tidal galaxy tails or galaxies which have hosted multiple $\mathrm{SNe}$.

The breakdown of the targets from the various feeder surveys is shown in Fig. 1 for the first year, which covers the first public data release. PESSTO formally collaborates with the LSQ Low Redshift Supernova Survey (Baltay et al. 2013) which operates as part of the La Silla-QUEST Southern Hemisphere Variability Survey (Baltay et al. 2012). The 160-megapixel QUEST camera is installed on the ESO Schmidt telescope, providing a sensitive pixel area of 8.7 square degrees and it uses almost all of the Schmidt telescope time. The pixel scale is $0{ }^{\prime} 88$ and the system gets to a depth of around $20^{\mathrm{m}}$ in 60 s through a wide-band filter that covers the SDSS $g+r$-bands. This magnitude limit is well matched to the capability of the NTT+EFOSC2 for obtaining spectra with signal-to-noise of greater than 10 in typically $20 \mathrm{~min}$ exposures. The LSQ survey repeats a sky area twice per night to remove bogus objects and asteroids, and in this way 1500 square degrees is typically covered each night. Figure 1 shows the source of targets for PESSTO classification from April 2012-2013 illustrating the extensive use of the LSQ survey targets. The Catalina Real-Time Transient Survey (CRTS Drake et al. 2009) is another very useful source of targets for PESSTO, as again the survey depths are well matched to PESSTO spectroscopic capabilities and the wide-field searching produces large numbers of transients that can be filtered for objects at the extreme end of the supernova luminosity distribution (e.g. Drake et al. 2010; Pastorello et al. 2010; Inserra et al. 2013) and luminous transient events in the cores of galaxies (Drake et al. 2011). PESSTO also parses the OGLE-IV transient list which is very useful as the declination of the fields are around -60 to -80 degrees, allowing the NTT to point in this direction during wind restrictions and providing targets available for long observational seasons. The other sources of targets as seen in Fig. 1 are the amateur reports which are posted on the Central Bureau's "Transient Objects Confirmation Page" 3 , the Chilean Automatic Supernova Search (CHASE; Pignata et al. 2009) and a small number of targets from MASTER (Lipunov et al. 2010) and TAROT (Klotz et al. 2008). Targets from the $3 \pi$ survey from Pan-STARRS1 survey have also been classified, mostly during the second year of operations (Smartt et al. 2014). In the future, PESSTO plans further exploitation of targets from the SkyMapper survey (Keller et al. 2007). Although we have a formal partnership and agreement to access the SkyMapper targets as soon as they are discovered, this survey was not functioning in full science survey mode during the period covered by the data in this paper. We also intend to exploit the transient stream likely to be produced by the ESA Gaia mission ("Gaia alerts", see Hodgkin et al. 2013).

One of the goals of PESSTO is to provide early spectra for both fast classification and for probing the early explosions of supernovae. The earlier an object can be classified, the more opportunity there is for the community to observe it with multiwavelength facilities in the interesting early phases of a few days after explosion (e.g. Soderberg et al. 2008; Cao et al. 2013) To gauge how the first year of PESSTO progressed, we compare the phases of the first classification spectra taken by PESSTO of La Silla-QUEST targets, with the Palomar Transient Factory

http://www.cbat.eps.harvard.edu/unconf/tocp.html 


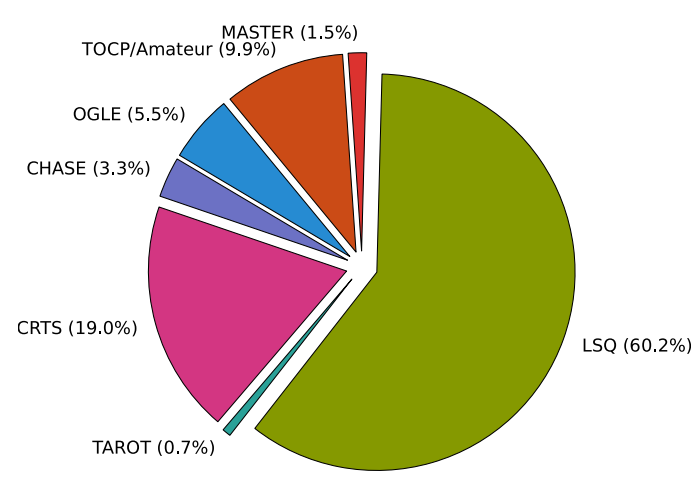

\begin{tabular}{lcc}
\hline Type & Number & Per Cent \\
\hline Ia & 130 & 47 \\
Ia-pec & 16 & 6 \\
II & 39 & 14 \\
IIn & 21 & 8 \\
IIb & 2 & 1 \\
Ib & 2 & 1 \\
Ic & 11 & 4 \\
SN uncertain & 5 & 2 \\
AGN & 3 & 1 \\
galaxy & 13 & 5 \\
stellar & 11 & 4 \\
unknown & 10 & 3 \\
not visible & 11 & 4 \\
Total & $\mathbf{2 7 4}$ & $\mathbf{1 0 0}$ \\
\hline
\end{tabular}

Fig. 1. Left: breakdown of the source of targets for PESSTO classifications from April 2012 to April 2013. The details of the survey names are in the text in Sect. 2.1. Right: classification types.
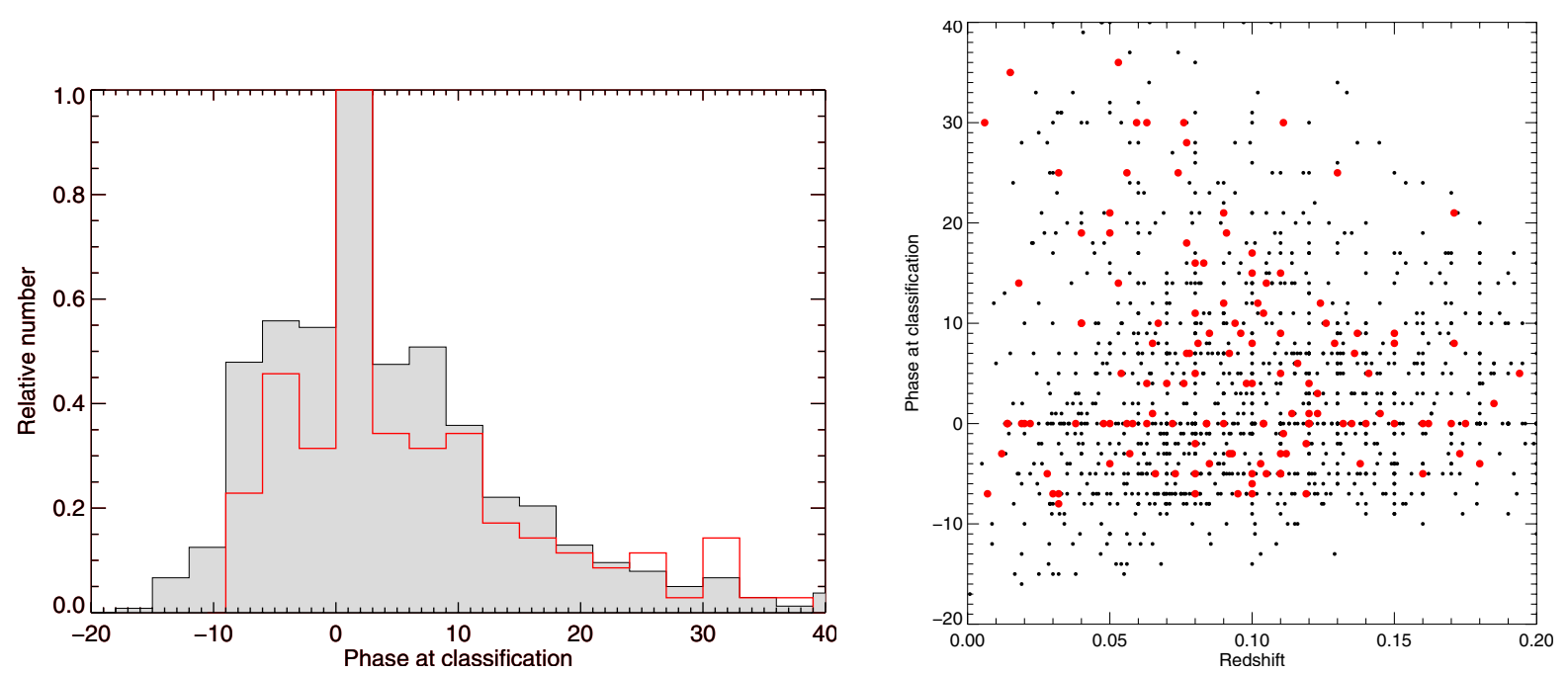

Fig. 2. Phase of type Ia SNe at first spectrum taken with PESSTO. Results from the Palomar Transient Factory (over 2009-2012) are in grey or black (data from Maguire et al. 2014), with PESSTO and LSQ targets in red.

in Fig. 2 (Maguire et al. 2014). We chose type Ia SNe for this comparison as they have well defined rise times, are the most common events found in magnitude limited surveys and can be typed to within a few days (type II SNe are hard to date from explosion, since the rise time takes hours to days if the progenitor is an extended red supergiant, and the peak is not a well defined epoch in this case). This shows that we recover type Ia SNe down to around 10 days before peak. There is some uncertainty in the dating of spectra as this epoch, but it is encouraging to see that we can recover SNe competitively at these epochs by combining immediate exchange of information between LSQ and PESSTO. There is an obvious peak in both surveys at phase $=0$ which is simply a labelling effect. Observers tend to label $\mathrm{SNe}$ which are close to peak magnitude as being "at peak" and therefore having phase $=0$. Spectroscopically dating type Ia $\mathrm{SNe}$ which are within a few days of peak magnitude is not accurate to within about $\pm 3-5$ days. Hence there is a human tendency to label these with phase $=0$. With the hindsight of lightcurves one can of course pin down the maximum light phase and then the true date of the first spectrum, but the phases plotted here typically come from spectroscopic dating only. In reality this peak should be smeared out within about \pm 5 days around the phase $=0$ epoch. Where PESSTO could do better, and where PTF has excelled, is in very low redshift early discoveries i.e. the bottom left corner of the right hand panel in Fig. 2. This is an area for rich scientific exploitation (e.g. Nugent et al. 2011; Gal-Yam et al. 2014). PESSTO publicly releases the first spectrum of each newly classified transient object it observes in reduced, flux calibrated form via WISeREP ${ }^{4}$ (Yaron \& Gal-Yam 2012). The raw data for every PESSTO observation are immediately available to the public once they are taken, via the ESO Archive. This includes the raw data for all classification spectra, imaging (including acquisition images), follow-up spectra and all calibration frames. The only delay is the time taken to copy data from La Silla and ingest into the ESO Garching archive which is typically minutes to hours. We release final reduced data of the science follow-up targets during yearly official data releases, the first of which is SSDR1.

\subsection{PESSTO Data reduction pipeline}

PESSTO uses fixed set-ups for EFOSC2 and SOFI which allow reduced data products to be provided rapidly and uniformly to the PESSTO survey members and the public (see Table 1 and 5 for the fixed set-ups). The PESSTO consortium has developed a data reduction pipeline, written in python by Stefano Valenti, with input from other members and based on the basic python packages numpy, pylab, pyraf, pyfits. The pipeline produces

4 http://wiserep.weizmann.ac.il 
Table 1. PESSTO settings for EFOSC2 spectroscopy.

\begin{tabular}{cllllllllll}
\hline \hline Grism & $\begin{array}{l}\text { Wavelength } \\
(\AA)\end{array}$ & $\begin{array}{l}\text { Filter } \\
\text { (blocking) }\end{array}$ & $\begin{array}{l}n_{\text {pix }} \\
(\text { pixels })\end{array}$ & $\begin{array}{l}\text { Dispersion } \\
\left(\AA \text { pix }^{-1}\right)\end{array}$ & $\begin{array}{l}F W H M \\
(\text { pixels })\end{array}$ & $\begin{array}{l}\text { Resolution } \\
(\AA)\end{array}$ & $\begin{array}{l}R \\
\left(\lambda_{c} / \Delta \lambda\right)\end{array}$ & $\begin{array}{l}V \text { resolution } \\
\mathrm{km} \mathrm{s}^{-1}\end{array}$ & $\begin{array}{l}\text { Arclines } \\
(\text { number })\end{array}$ & $\begin{array}{l}\text { rms } \\
(\AA)\end{array}$ \\
\hline$\# 13$ & $3650-9250$ & none & 1015 & 5.5 & 3.3 & 18.2 & 355 & 845 & $13-15$ & $0.10-0.15$ \\
$\# 11$ & $3345-7470$ & none & 1011 & 4.1 & 3.4 & 13.8 & 390 & 765 & 9 & $0.10-0.15$ \\
$\# 16$ & $6000-9995$ & OG530 & 950 & 4.2 & 3.2 & 13.4 & 595 & 504 & $11-14$ & $0.05-0.10$ \\
\hline
\end{tabular}

Notes. The number of pixels and wavelength ranges are those in the final trimmed spectra for public release. The blocking filter OG530 is used only (and always) for Gr\#16. The spectral resolution $R$ is given at the central wavelength, as is the velocity resolution $V$. The FWHM is the full-width-half-maximum of a Gaussian fit to either the [O I] $5577 \AA$ or $6300 \AA$ night sky line, for a 1". 0 slit. The respective values when a 1.5 slit is used can be determined by simply multiplying the values by 1.5 . The column headed Arclines indicates the number of lines used. The rms is the typical residual for the wavelength calibration solution.

fully reduced, flux calibrated spectra (1D and 2D images) for both EFOSC2 and SOFI and reduced images for EFOSC2 and SOFI on which photometric measurements can be made (a nominal zeropoint is provided as discussed below). This pipeline is publicly available on the PESSTO wiki, with instructions for installation and use. All the data released in SSDR1 have been processed through the pipeline and indeed all subsequent data releases will be similarly processed. The following sections describe the instrument set-ups, calibrations and data products delivered including details on the header keywords employed that assist the user in interpreting the calibrated data.

In the first year of PESSTO and for SSDR1, we have not focused on high accuracy absolute spectrophotometry. Reliable relative flux calibration is essential for supernovae and transient science, but for general classification and screening absolute spectrophotometry is not critical. We placed more importance on screening and classifying as many targets as possible rather than higher accuracy calibration of fewer objects. For follow-up targets, the standard methods of improving spectrophotometry involve correcting the spectra with photometric measurements from time series lightcurves. Since most of the PESSTO science targets do not yet have a fully calibrated lightcurve, we have not corrected the spectra in bulk with this method. Discussion of the accuracy and reliability of the absolute flux scales is presented in Sect. 3.3 and future data releases will focus on improving this.

\section{PESSTO EFOSC2 spectroscopic observations and calibrations}

The ESO Faint Object Spectrograph and Camera 2 (EFOSC2) has been mounted at the $f / 11$ nasmyth focus on the NTT since 2008. It is a focal reducer which uses multi-layer coated, alltransmission optics (i.e. no reflecting surfaces). The F/49 camera has a focal length of $200 \mathrm{~mm}$, which provides a pixel scale of 0 .' $^{\prime} 12$ pix $^{-1}$, for detector pixels of $15 \mu \mathrm{m}$ physical size. As described in the EFOSC2 manual, the filter and grism wheels are located in the parallel beam, between the collimator and the camera which means that the EFOSC2 focus is quite stable and does not drift significantly as a function of temperature, rotator angle, wavelength or observing mode.

As an all-transmission optical instrument, the dispersing elements are grisms, providing a fixed wavelength coverage dependent on detector size and camera beam width. Despite the large number of grisms available, PESSTO uses only three default settings for EFOSC2, listed in Table 1. The CCD on EFOSC2 is ESO\#40 which is a Loral Lesser Thinned AR coated, UV flooded detector with $2048 \times 204815 \mu$ m pixels

\footnotetext{
http://www . eso.org/sci/facilities/lasilla/

instruments/efosc.html
}

and driven with an ESO-FIERA controller. PESSTO uses the CCD in Normal readout mode with $2 \times 2$ pixel binning giving 2-dimensional science images with $1024 \times 1024$ physical pixels, which have a plate scale of 0.24 pixel $^{-1}$. The CCD is never windowed for PESSTO observations, hence the field size is $4.1 \times 4.1$ arcmin. The lowest resolution setting $(\mathrm{Gr} \# 13)$ of $17.7 \AA$ has the broadest wavelength coverage and is the default setting for classification spectra (Table 1). All classification spectra which are reduced and released immediately (within $24 \mathrm{~h}$ of the data being taken) employ this set-up. The other two grism settings are used for some of the PESSTO Key Science Targets, in which either the higher resolution or full wavelength coverage (or both) are required. All PESSTO observations with Gr\#16 use the order blocking filter OG530 to remove second-order effects in this red setting. For science targets PESSTO does not use an order blocking filter for Gr\#13. Hence for blue objects there may be second-order contamination at wavelengths greater than $>7400 \AA$. In order to correct for this, spectrophotometric flux standards are taken with and without a blocking filter; further details on how this correction is applied are in Sect. 3.3. The EFOSC2 aperture wheel has fixed width slits. When the seeing is $\leq 11^{\prime \prime} 4$ then the $1^{\prime \prime} .0$ slit is used and when it is $\geq 1$.' 5 then then the 1".5 slit is employed. Flat field and flux calibrations are then taken with the appropriate spectrograph configuration and matched to the science frames within the data reduction pipeline. The spectrograph set-ups are summarised in Table 1.

\subsection{Detector characteristics : bias level, gain and readnoise}

The EFOSC2 chip, CCD\#40, is always used by PESSTO in Normal readout mode and $2 \times 2$ binning (Mode 32 as defined by $\mathrm{ESO}^{6}$ ). All acquisition images are also taken in this mode. At the beginning of the PESSTO survey and during April 2012, we began with the EFOSC2 default acquisition Observation Blocks (OBs) which use Fast readout mode for acquisition images. This is immediately visible to the user as Fast readout mode uses two amplifiers and the frame shows a split appearance with two halves of the chip having different bias levels. This readout mode was never used for PESSTO science frames and from August 2012 onwards, PESSTO has uniformly used Normal readout mode for all acquisition frames (except in a few occasions because of $\mathrm{OB}$ selection error). In this section we present characterisation checks of the CCD only in Mode 32: Normal readout mode and $2 \times 2$ binning. The CCD has a physical size of $2048 \times 2048$ pixels. With 12 pixels of prescan and overscan this results in a $2060 \times 2060$ pixel FITS image, or a $1030 \times 1030$ pixel FITS file after binning. Hereafter, all pixel

\footnotetext{
6 http://www.eso.org/sci/facilities/lasilla/

instruments/efosc/inst/Ccd40.html\#Performance
} 




Fig. 3. Bias level of CCD\#40 over the first year of PESSTO survey operations. Red pluses are the mean count as measured over the central $200 \times 200$ binned pixels in each raw bias frame. Black crosses are the mean of each combined masterbias frame produced by the PESSTO pipeline from a set of raw biases, and measured over the same region. Error bars correspond to the standard deviation of the measured pixels, while the solid line is the average over the year.

coordinates referred to will be on this $1030 \times 1030$ reference frame.

The bias level of CCD\#40 on EFOSC2 appears to be stable to within $5-10 \%$ of the mean level (212 ADU) across a period of a year. In Fig. 3 we plot the bias level from all PESSTO nights during the first year of survey operations. This was measured in the central $200 \times 200$ binned pixel region. The bias frames are always flat and uniform and no evidence of gradients have been found. Hence no overscan region corrections are ever applied in the PESSTO pipeline processing. The EFOSC2 Users Manual (Monaco et al. 2012) notes that overscan correction should not be used owing to their small size (6 and 12 pixels only) and bleeding effects of charge from the science and calibration frames into these sections.

To measure the gain and readout noise of CCD\#40, we used the FINDGAIN task within IRAF. FINDGAIN uses Janesick's algorithm to determine the gain and readout noise of a CCD from a set of two bias frames and two frames with uniformly high signal levels. We used pairs of EFOSC2 dome flats from the ESO archive (in the V\#641 filter and Mode 32) during the months of PESSTO observations. We selected two exposures from each set of domeflats, and used the closest bias frames available (usually from the same night, but occasionally from the preceding or subsequent night) and ensured that all were taken in Mode 32 (i.e. Normal readout mode and $2 \times 2$ binning). The gain and readnoise were measured over the region of the CCD from [601:800, 401:600] (in binned pixels) to minimise the effects of any slope in the flat field. Sixty five such measurements were made over the period of PESSTO observations from April 2012 to April 2013 and the results are plotted in Fig. 4. The gain was found to be stable, with a mean value of $1.18 \pm 0.01 \mathrm{e}^{-} / \mathrm{ADU}$. The readout noise appears to be constant until January 2013, when it increased from an average of $\sim 11 \mathrm{e}^{-}$to $\sim 12.5 \mathrm{e}^{-}$. The cause of the readout noise increase is not known to us, but our measurements match those of the ESO La Silla Quality Control programme which also records an increase in the noise in this readout mode.

As described in the EFOSC2 instrument handbook the bad pixel map of CCD\#40 (see also the EFOSC2 BADPIXMASK ${ }^{7}$ ) indicates a bad column at $X=486$ (in binned image coordinates). The dispersion direction runs along the $Y$-direction, and

\footnotetext{
7 http://www.eso.org/sci/facilities/lasilla/ instruments/efosc/inst/BADPIXMASK.html
}

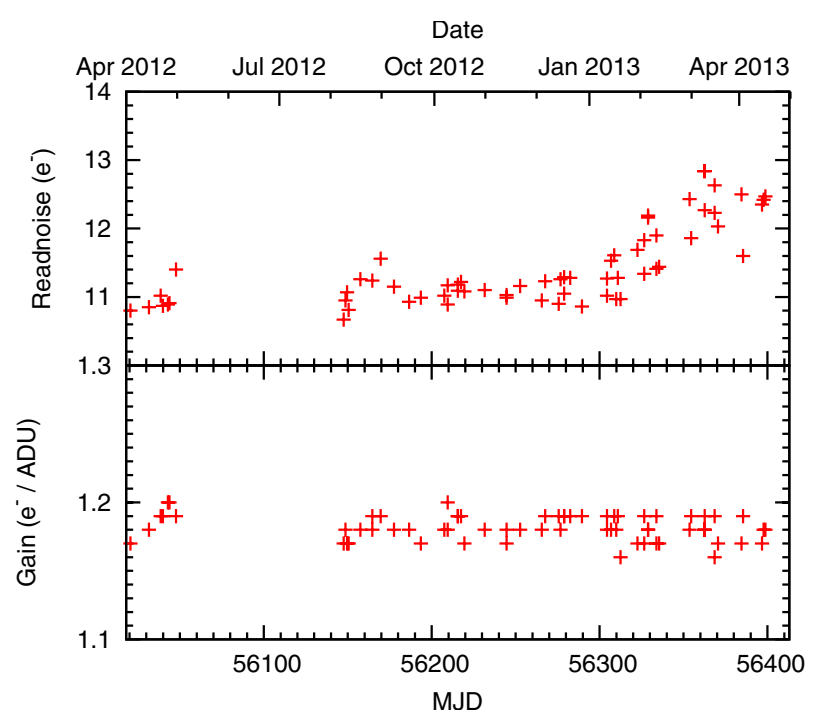

Fig. 4. Readnoise and gain of CCD\#40 over the period of the first year of PESSTO survey observations. The method to determine the values is described in the text.

hence as is standard practice for EFOSC2 observing, PESSTO targets are positioned at $X=550$. To be clear, these $X$-positions refer to the CCD pixel coordinates in the full raw $1030 \times$ 1030 frame (i.e. including the prescan and overscan sections). After processing, the PESSTO data products are trimmed to 851 pixels in the spatial direction and the targets are typically at pixel $X=450$.

\subsection{Spectroscopic calibration data and reduction}

PESSTO observations are carried out in visitor mode and the project aims to homogenise all calibration frames and tie these directly to what is required in the data reduction pipeline. To achieve that, standard sets of OBs for calibration purposes have been created, and are available on the PESSTO public wiki. Here we describe the calibration data that are taken during routine PESSTO observing and how they are applied in the data reduction process.

\subsubsection{Bias calibration}

As discussed above in Sect. 3.1 the bias level has been measured to be quite stable over a one year period. A set of 11 bias frames are typically taken each afternoon of PESSTO EFOSC2 observations and are used to create a nightly master bias. This nightly master bias frame is applied to all EFOSC2 data taken, including the spectroscopic frames, the acquisition images and any photometric imaging. The frame used for the bias subtraction can be tracked in the header keyword

ZEROCOR = 'bias_20130402_Gr11_Free_56448.fits'

The file name gives the date the bias frames were taken, the Grism and filter combinations for which it is applicable (of course for biases this is not relevant but the pipeline keeps track with this nomenclature) and the MJD of when the master bias was created. The dark current is less than $3.5 \mathrm{e}^{-} \mathrm{pix}^{-1} \mathrm{~h}^{-1}$, hence with typical PESSTO exposures being 600-1800s, no dark frame correction is made. 


\subsubsection{Flat field calibration}

Techniques for flatfielding spectroscopic data depend on the particular instrument or detector response that is being targeted for removal. The type of calibration data required needs to be tailored to the process and the final science product requirement.

The PESSTO survey takes sets of spectroscopic flatfields in the afternoons at a typical frequency of once per sub-run of 3-4 nights. The illumination for these comes from outside the instrument, hence they are referred to as "spectroscopic dome flats". An integrating sphere is illuminated with a "flatfield" lamp (a tungsten halogen with a quartz bulb) which is directed towards the NTT focal plane and the telescope pupil is approximately simulated. The EFSOC 2 calibration OBs allow the user to set a required level of counts. PESSTO takes five exposures with maximum count levels of 40000-50000 ADU for each of the grism, order sorting filter, and slit width combinations that we use. There are 8 combinations in total: the three basic configurations as listed in Table 1, which are used with 1 and 1.5 arcsec slits and in addition Gr\#13 flats are taken with the GG495 filter, to allow for second-order correction as discussed in Sect. 3.3. Each of these is combined and normalised to give a masterflat which can be associated with the appropriate science observations from the sub-run. This is recorded in the FITS header for each data product, for example:

FLATCOR = 'nflat_20130413_Gr11_Free_slit1.0_100325221_56448.fits'

As these are primarily used for removal of pixel-to-pixel response then higher frequency observations are not necessary. In fact it is debatable as to whether pixel-to-pixel response removal is required at all in long-slit spectroscopy at moderate signal-to-noise. PESSTO does not take, nor use, spectroscopic sky flats during twilight for correction for slit illumination patterns. As we are primarily concerned with flux calibration of point sources, and the spectroscopic standard is placed at the same position on the slit and detector as the science targets (at CCD pixel position $X=550$ ) then application of a slit illumination correction is not necessary.

The EFOSC2 CCD\#40 is a thinned chip, hence has significant fringing beyond $7200 \AA$ and the severity depends upon the grating used. To remove this fringing pattern (in spectroscopic mode) a calibration flat field lamp exposure immediately after or before the science image is required and is divided into the science spectrum (another method is to construct fringe frames directly from on-sky frames during the night, but this requires that the targets are dithered along the slit; as discussed in Ellis et al. 2008). Afternoon flats are not useful for this owing to wavelength drifts that alter the fringe pattern and hence do not allow for removal. PESSTO always takes internal lamp flats (3 exposures of typically 40000 ADU maximum count level) after taking any science spectra with Gr\#16. We do not, as default, take fringe correcting flats when using Gr\#13, as the fringing does not affect this grism to a significant extent, as can be seen in Fig. 5. The internal lamp in EFOSC2 is a quartz lamp, which suffers from water vapour absorption that is not present in the afternoon dome flats.

In all cases, the combined flats are normalised to remove their overall shape, while leaving the pixel to pixel response (and in the case of Gr\#16, the fringing pattern) that we are attempting to correct for. High-order splines are fitted to the flat fields, with orders 90, 35 and 70 for Gr\#13, Gr\#11 and Gr\#16 respectively. In Fig. 6 we show the shape of the flat fields used by PESSTO, both before and after normalisation. In the pixel regions below 200 for Gr\#11 (which is approximately $4450 \AA$ ), the

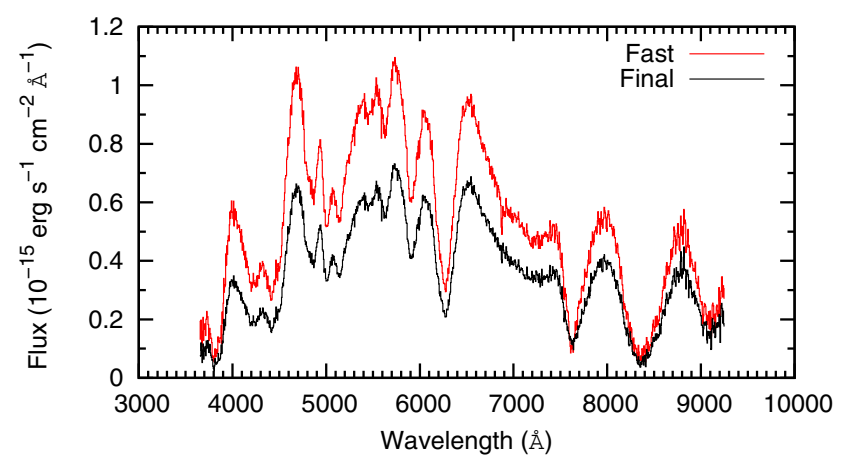

Fig. 5. Comparison of the rapid and final reductions for the classification spectrum of SN 2012fx, taken with Gr\#13 and a 1" slit on 2012 August 26. These spectra show that fringing is not as significant a problem with Gr\#13 as it is for Gr\#16.

response of the dome flat field lamp is very poor, resulting in low signal. Even after combining the frames, the count rate is typically $1700 \mathrm{e}^{-}$which would only increase the noise in the science frames rather than improve it. Hence we set the flat level to unity between pixels 1 and 200. The same effect occurs for Gr\#13 and we do not flat field the first 200 pixels of each spectra. In summary, for Gr\#11 and Gr\#13 we employ masterflats constructed using the dome lamps for each sub-run of 3-4 nights. For Gr\#16 we do not use masterflats, but instead use three flatfields taken with the internal lamp immediately after the science spectrum is taken. Figure 6 illustrates the profile of the normalised flats used in the flat-fielding process. It is noticeable that Gr\#11 is not flat at the unity level, but has variations of order $3-4 \%$. While this is not ideal, and will be fixed in future data releases (e.g. SSDR2), we have left it as shown, as the variation will not affect the flux calibration since both the standards and science objects are treated with the same flat-field. We also note that the normalisation of the dome flats often is not continuous at the point where we set it to unity and discrete steps are induced at the level of 1-3\% (the examples shown in Fig. 6 for Gr\#11 is one of the worst cases). One might expect this to be propagated into the science spectra since the response functions derived from the spectrophotometric standards will not reproduce such a step function. We have checked the highest signal-to-noise science spectra and don't see obvious signatures of this flat-field feature. Nevertheless it is an undesirable feature of the released dataset and we will aim to fix in the next data release.

Figure 7 shows how fringing is almost completely removed in Gr\#16 spectra using an internal contemporaneous flat-field. It also illustrates that using a flat from the afternoon (not at the same telescope position) has no impact on fringe removal, and in fact makes it slightly worse.

\subsubsection{Cosmic ray removal}

EFOSC2 spectra with a typical exposure time of $\sim 1800 \mathrm{~s}$ will show numerous cosmic ray hits in the $2 \mathrm{D}$ frames, as can be seen in the upper panel of Fig. 8. After de-biasing and flatfielding, we use the cosmic ray rejection algorithm LACOSMIC ${ }^{8}$ presented in van Dokkum (2001) to remove these. The PESSTO pipeline incorporates a modified version of the python implementation ${ }^{9}$ of LACOSMIC that avoids the use of the scipy package. As it is a computationally expensive process during the

\footnotetext{
8 http://www.astro.yale.edu/dokkum/lacosmic/

9 http://obswww.unige.ch/\$\sim\$tewes/cosmics_dot_py/
} 


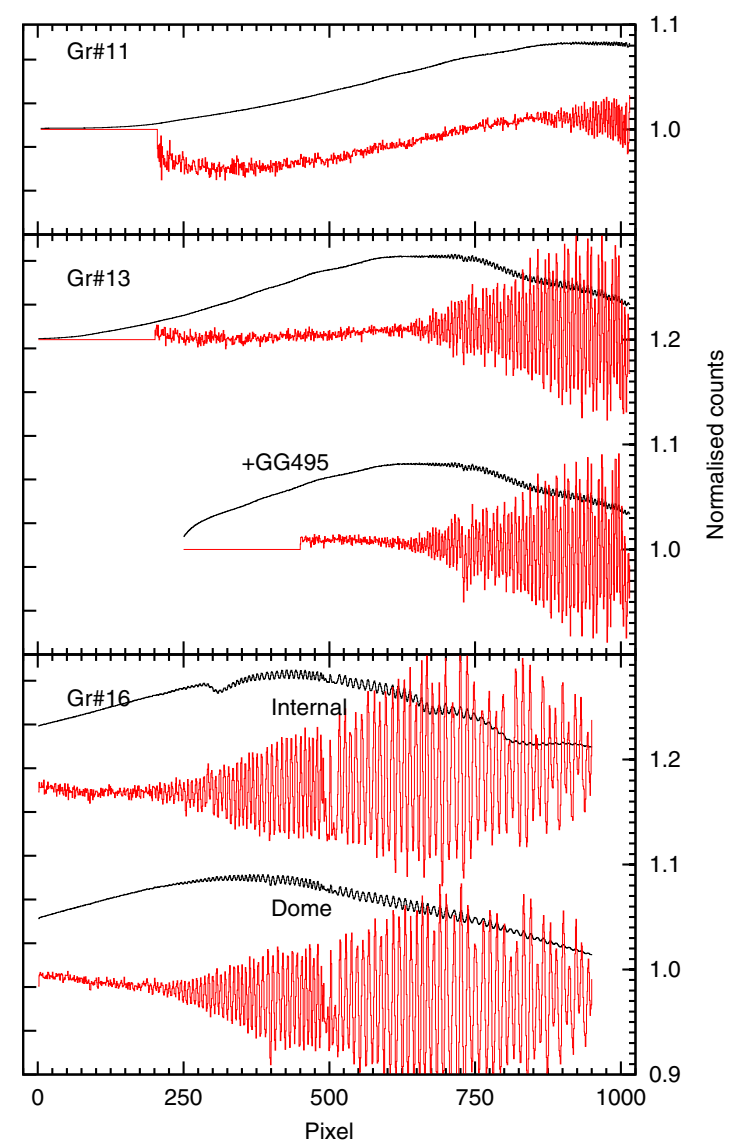

Fig. 6. Representative flat fields for Gr\#11, 13 and 16, taken in August 2013. Each panel shows a cut along column 450 of the twodimensional flat-field, corresponding to the approximate pixel coordinates of the target. The black lines show the intrinsic shape of the flat fields, while the red lines show the normalised flat field after fitting with a high-order polynomial (all spectra are normalised to 1, and offset for clarity). For Gr\#13, the flat field with the GG495 order blocking filter (as used when correcting for second-order contamination) is also shown. For Gr\#16, both internal flats and dome flats are shown; in the former the absorptions due to $\mathrm{H}_{2} \mathrm{O}$ vapour are visible in the unnormalised flat. This absorption feature is fit by the high-order polynomial during normalisation and hence is removed in the normalised flat.

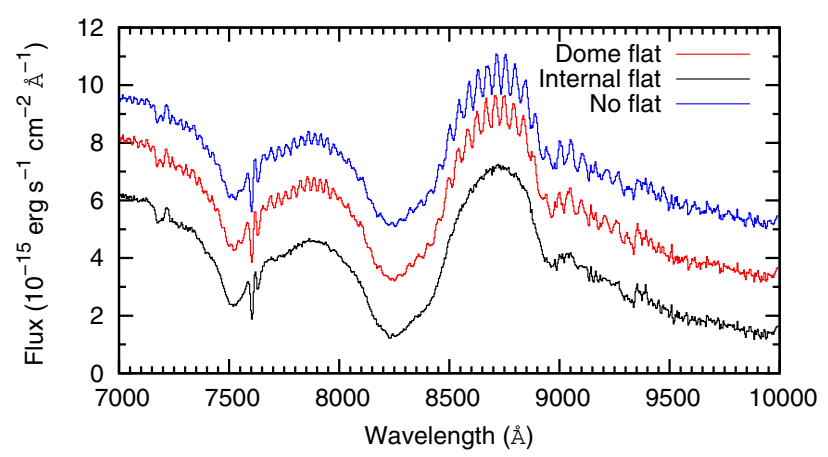

Fig. 7. Gr\#16 spectrum of SN 2011 gr. Three reductions are shown, in the first there is no flat-fielding, in the second a dome flat from the start of the night is used, in the third an internal flat taken immediately after the science observations.

manual extraction, only the central 200 pixels around the object are cleaned (i.e. central pixel \pm 100 pixels). Figure 9 shows an example of the extracted spectrum before and after cosmic ray cleaning. One concern of applying this cosmic ray rejection is whether it may erroneously remove real, narrow,

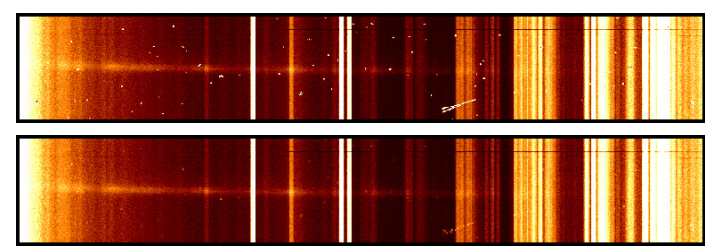

Fig. 8. 2D spectrum of LSQ12drz, reduced with and without LACOSMIC applied. The PESSTO pipeline applies this, but only in the central \pm 100 pixels around the science object.

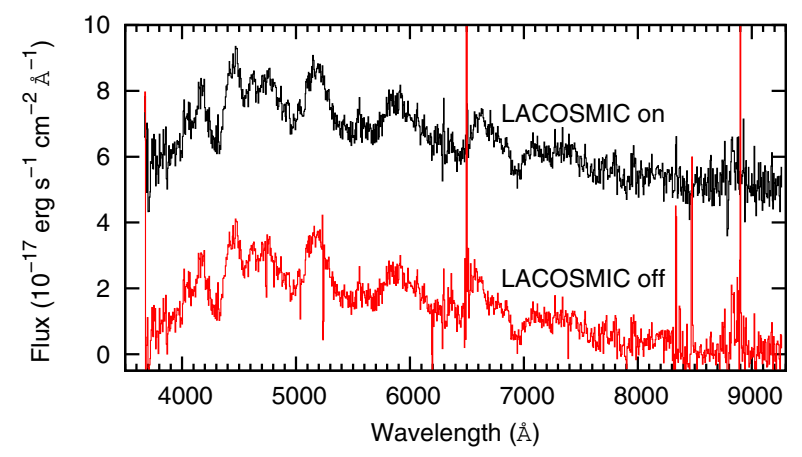

Fig. 9. Classification spectrum for LSQ12drz taken on 2012 August 7, reduced with PESSTOFASTSPEC with and without cosmic ray rejection. The relatively long exposure time for this spectrum (2700 s) results in a large number of cosmic ray hits, which are removed in the $2 \mathrm{D}$ image using the algorithm of van Dokkum (2001).

emission features from spectra. However, we have tested this in the EFOSC2 data, and in particular for SN 2009ip which has narrow lines, and are confident that this is not the case. Whether cosmic rays were removed or not is recorded in the header as described in Sect. A.2.

\subsubsection{Arc frames and wavelength calibrations}

Arc frames are generally taken in the evening before observing and are never taken during dark time. EFOSC2 has helium and argon lamps and PESSTO uses both of these lamps turned on together. No arc frames are taken during the night to reduce overheads. Although EFOSC2 suffers from significant flexure as the instrument rotates at the nasmyth focus (which can be 4 pixels over 200 degrees in rotation), the flexure causes a rigid shift of the wavelength frame. Hence we apply the calibration determined from the evening arc frames and adjust this with a linear offset as measured from either the skylines or atmospheric absorption lines.

Relatively high-order Legendre polynomial fits are needed to fit the EFOSC2 arc lines with a fit which produces no systematic residuals. For Gr\#13, 13-15 lines were used with a fifth- or sixth-order fit; when the GG495 order blocking filter was also used the order of the fit was reduced to 5 (due to the smaller wavelength range). For Gr\#11, 9 lines were used with a fifthorder fit, while for Gr\#16 11-14 lines were used for a fifth or sixth-order fit. The root mean square (rms) error of the fit was typically found to lie between 0.1 and $0.2 \AA$ as shown in Fig. 10. The number of arc lines used for the dispersion solution of each object, along with the rms error, are given in the header of the reduced spectra by the keywords LAMNLIN and LAMRMS respectively. The formal rms values are probably too small to realistically represent the uncertainty in the wavelength calibration at any particular point, given the FWHM of the arclines is $13-17 \AA$. Hence this might suggest over-fitting of the 
S. J. Smartt et al.: PESSTO: survey description and data products

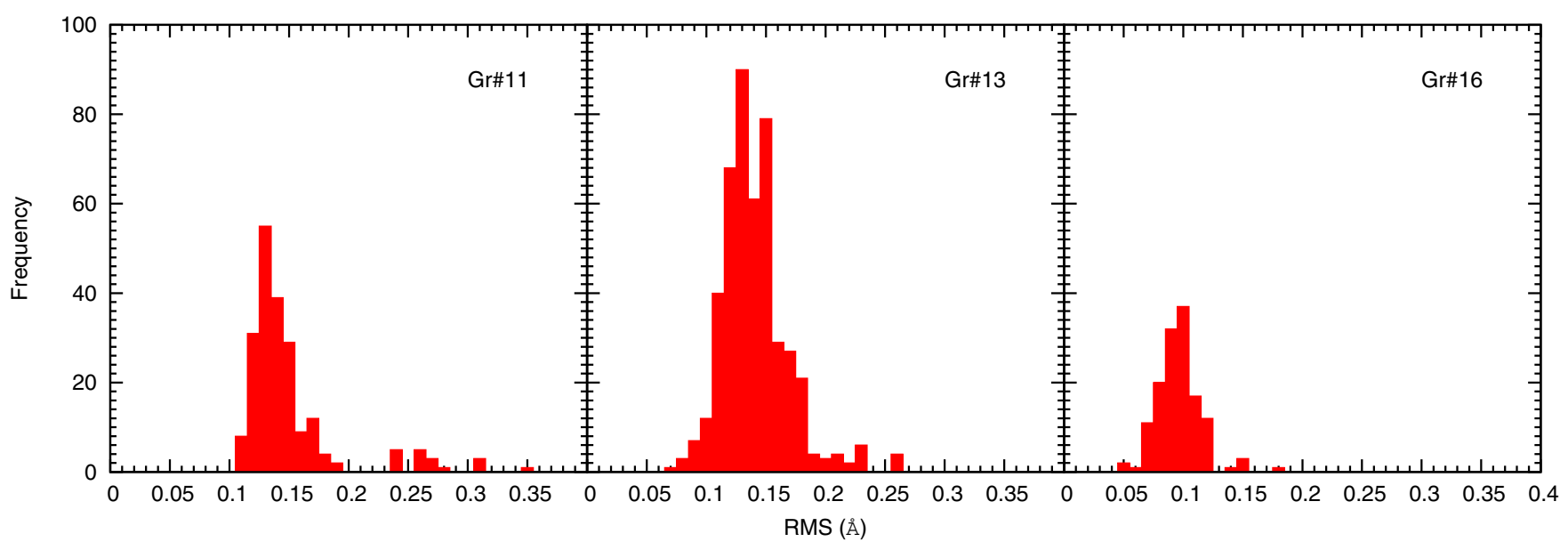

Fig. 10. Histogram of the root mean square error of the fit to the arc lines for each science spectrum. Each panel includes $1.0^{\prime \prime}$ and $1.5^{\prime \prime}$ slit spectra.

Table 2. PESSTO spectrophotometric standards.

\begin{tabular}{lcccccl}
\hline \hline Standard name & RA (FK5, J2000) & Dec (FK5, J2000) & Proper motion $(\mathrm{mas} / \mathrm{yr})$ & $V$ mag & Sp. Type & Instrument \\
\hline VMA2 & 004909.902 & +052319.01 & $1236.90,-2709.19$ & 12.374 & DZ8 & EFOSC2 \\
GD71 & 055227.614 & +155313.75 & $85,-174$ & 13.032 & DA1 & EFOSC2/SOFI \\
L745-46a & 074020.79 & -172449.1 & $1129.7,-565.7$ & 12.98 & DAZ6 & EFOSC2 \\
LTT 3218 & 084132.50 & -325634.0 & $-1031.7,1354.3$ & 11.85 & DA5 & SOFI \\
LTT3864 & 103213.603 & -353741.90 & $-263.7,-8.0$ & 11.84 & Fp $\ldots$ & EFOSC2 \\
GD153 & 125702.337 & +220152.68 & $-46,-204$ & 13.35 & DA1.5 & EFOSC2/SOFI \\
EG274 & 162333.837 & -391346.16 & $76.19,0.96$ & 11.029 & DA2 & EFOSC2/SOFI \\
EG131 & 192034.923 & -074000.07 & $-60.87,-162.15$ & 12.29 & DBQA5 & EFOSC2 \\
LTT 7379 & 183625.941 & -441836.93 & $-177.05,-160.31$ & 10.22 & G0 & EFOSC2 \\
LTT 7989 & 201112.08 & -360606.5 & $522,-1691$ & 11.5 & M5V & SOFI \\
Feige110 & 231958.398 & -050956.16 & $-10.68,0.31$ & 11.5 & sdO & EFOSC2/SOFI \\
\hline
\end{tabular}

Notes. All data in this table are taken from Simbad.

sampled points. As a comparison, Legendre polynomials with order 4 produced obvious systematic residuals and rms values of between $0.4-1.0 \AA$ for a 1 .'.0 slit and 1-1.8 $\AA$ for a 1 '.'5 slit.

For exposures longer than $300 \mathrm{~s}$, the linear shift applied to the dispersion solution is measured from the night sky emission lines. For shorter exposures (brighter objects), the night sky lines are weak or not visible, and the shift is instead measured from the telluric absorptions in the extracted 1D spectrum. The linear shifts are calculated by cross-correlating the observed spectrum (sky or standard) with a series of library restframe spectra which are offset by $1 \AA$. The library spectrum which produces the minimum in the cross-correlation function is taken as the correct match and this shift is applied. This method limits the precision of the shift to $1 \AA$, which is roughly $\frac{1}{4}$ of a pixel and less than $\frac{1}{10}$ of a resolution element. This value of $1 \AA$ is recorded in the header as the systematic error in the wavelength calibration (SPEC_SYE, see Appendix A.3).

The value of the linear shifts applied are typically in the range of 6-13 $\AA$ for Gr\#11 and Gr\#13. In the case of Gr\#16 spectra the shifts were usually smaller, usually 4-9 $\AA$. This value is recorded in the header keyword SHIFT. Full details of the header keywords applicable for the wavelength solution are in Appendix A.3.

\subsection{Spectrophotometric standards and flux calibration}

PESSTO uses a set of 9 spectrophotometric standard stars for EFOSC2, listed in Table 2. The data in this table are taken directly from Simbad ${ }^{10}$. The EFOSC2 finding charts, including proper motion projections, are available for users from the PESSTO website, and the data tables used for flux calibration standards are available in the publicly accessible PESSTO pipeline. These standards provide year round coverage, have full wavelength coverage from the atmospheric cut-off up to $1 \mu \mathrm{m}$ and are in a suitable magnitude range for a $3.5 \mathrm{~m}$ aperture telescope. PESSTO standard policy is to observe an EFOSC2 spectrophotometric standard three times per night (start, middle and end), although if there are significant SOFI observations or weather intervenes then this may be reduced. Generally, the three observations will include 2 different stars and a set of observations is taken with all grism, slit and filter combinations used during the nights observing. From September 2012 to November 2012 the standard EG21 was frequently observed. We later realised however that the photometric flux tables for this star did not cover the full, telluric corrected regions for Gr\#16 and Gr\#13 and hence stopped using it after 2012-11-21. We have only used it to calibrate PESSTO data taken with Gr\#11 in SSDR1.

The wavelength coverage of GR\#13 is 3650-9250 $\AA$, and for science targets we do not use an order blocking filter. Hence second-order contamination is possible for blue objects beyond around $7200 \AA$, depending on their colour. This would also affect the flux standards and hence the flux calibration of science targets. To remove any second-order contamination in the flux standards, PESSTO always takes Gr\#13 data for these stars with and without the filter GG495, to allow correction for the

${ }^{10}$ http://simbad.u-strasbg.fr/simbad/ 

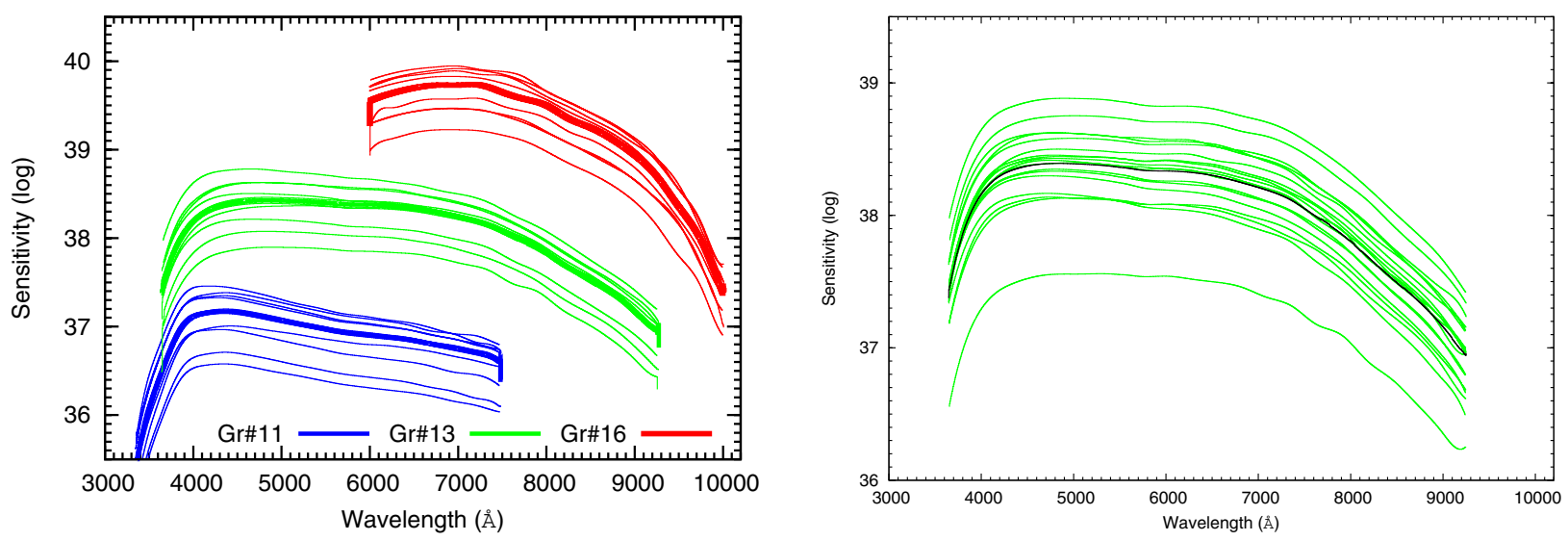

Fig. 11. Left: average monthly sensitivity curves for Gr\#11, Gr\#13 and Gr\#16, derived from spectrophotometric standards taken during the first year of PESSTO observations. The thick lines are annual sensitivity function. Thin lines are monthly averages (Gr\#11 offset by -1 , Gr\#16 offset by +1 for legibility) Right: as a comparison, the sensitivity curves from individual standard stars over ten nights in March 2013 (for Gr\#13). Four different standards were observed, L745a, LTT3864, EG274, GD71. The master sensitivity curve for March 2013 with this configuration is shown in black.

effect during pipeline reductions. The blocking filter GG490 has a transmission of $90 \%$ from $5000 \AA$ and upwards. The sensitivity function for the combination of Gr\#13+GG490 is scaled up to match the sensitivity function of Gr\#13+Free at the position of $5500 \AA$. To construct a final sensitivity function which is corrected for any second-order flux in the standards, we merge the sensitivity function Gr\#13+free (from 3650-5500 $\AA$ ) and the scaled up sensitivity function of Gr\#13+GG490 (for wavelengths $>5500 \AA$ ). Flux standards are always observed unless clouds, wind or humidity force unexpected dome closure. Hence even during nights that are not photometric, flux standards are taken and the spectra are flux calibrated; we deal with the issue of the absolute flux reliability below.

A sensitivity function is derived for each EFOSC2 configuration from the spectrophotometric standards observed. These were averaged to create a master sensitivity function for each month, which was then applied to the final reduced spectra. In a few instances, a master sensitivity curve was not created for a particular configuration on a given month, as there were no appropriate standards observed. In these cases, the sensitivity function from the preceding or following month was used. In Fig. 11, we show the shape of the sensitivity curves, together with the variation in sensitivity functions from month to month. The sensitivity curves from individual standard stars within a particular month are shown for comparison. While there are grey shifts between the individual sensitivity curves (as expected for observations taken with a single slit width under differing atmospheric conditions), the overall shape of the sensitivity curves are quite similar, indicating that the relative flux calibration is reliable.

The standard method of ensuring spectra are properly flux calibrated is to compare synthetic photometry of the science spectra with contemporaneous calibrated photometry and apply either a constant, linear or quadratic multiplicative function to the spectra to bring the synthetic spectra into line with the photometry. For PESSTO SSDR1 this is not yet possible for all spectra since the photometric lightcurves are not yet finalised for many of the science targets and the classification spectra do not have a photometric sequence. However it is useful to know what the typical uncertainty is in any flux calibrated PESSTO spectrum, and this is encoded in the header keyword FLUXERR. PESSTO observes through non-photometric nights, and during these nights all targets are still flux calibrated. Hence the uncertainties in flux calibrations come from transparency (clouds), seeing variations that cause mismatches between sensitivity curves derived using standards with different image quality, and target slit positioning. Finally, photometric flux is generally measured with point-spread-function fitting, which inherently includes an aperture correction to determine the total flux whereas spectroscopic flux is typically extracted down to 10 per cent of the peak flux (a standard practice in IRAF's apall task). All of this means that large variations are expected and we carried out tests as to how well this method works and what is the reliability of the absolute flux calibration in the spectra.

We took the Gr\#11, Gr\#16 and Gr\#13 spectra of the three targets for which a calibrated photometric sequence is either published or has been measured and is in preparation: SN 2009ip (Fraser et al. 2013) SN 2012fr (Childress et al. 2013, and Conteras et al., in prep.) SN 2013ai (Fraser et al., in prep). Synthetic BVRI photometry on the PESSTO spectra was calculated using the SYNPHOT package within IRAF and spectra which covered the entire bandpass of each filter were included and the difference between the spectral synthetic magnitudes and photometric measurements is shown in Fig. 12. This illustrates the difficulty and challenges faced in accurately flux calibrating spectra but also shows promise that in future data releases we can significantly improve on SSDR1. The standard deviation of all points in $\pm 0.31^{\mathrm{m}}$ or $\pm 29 \%$, but we can identify several cases were fairly obvious catastrophic failures have occurred. The main bulk of points lie within a range of $\pm 0.44^{\mathrm{m}}$ around zero, with a formal average offset and standard deviation of $0.042 \pm 0.164^{\mathrm{m}}$. The four low lying points have either images of poor seeing, when the seeing changed rapidly before the slit width was changed or (in the case of the point at -0.9 ) the object likely was not positioned on the slit. The points lying above +0.5 were found to be owing to a monthly sensitivity curve for January 2013 which was too low, likely due to an unusually high frequency of nonphotometric cloudy conditions when the standards were taken. Ignoring these fairly obvious problematic cases, the scatter in the flux calibration is $\sim 15 \%$ in the main locus and is recorded in the headers of all spectra.

FLUXERR $=15.2 /$ Fractional uncertainty of the flux $[\%]$

Science users should use this as a typical guide, if the seeing (as can be measured on the 2D frames and acquisition images) and night conditions (from the PESSTO wiki night reports and see Appendix B) are reasonable. Note that we have recorded 


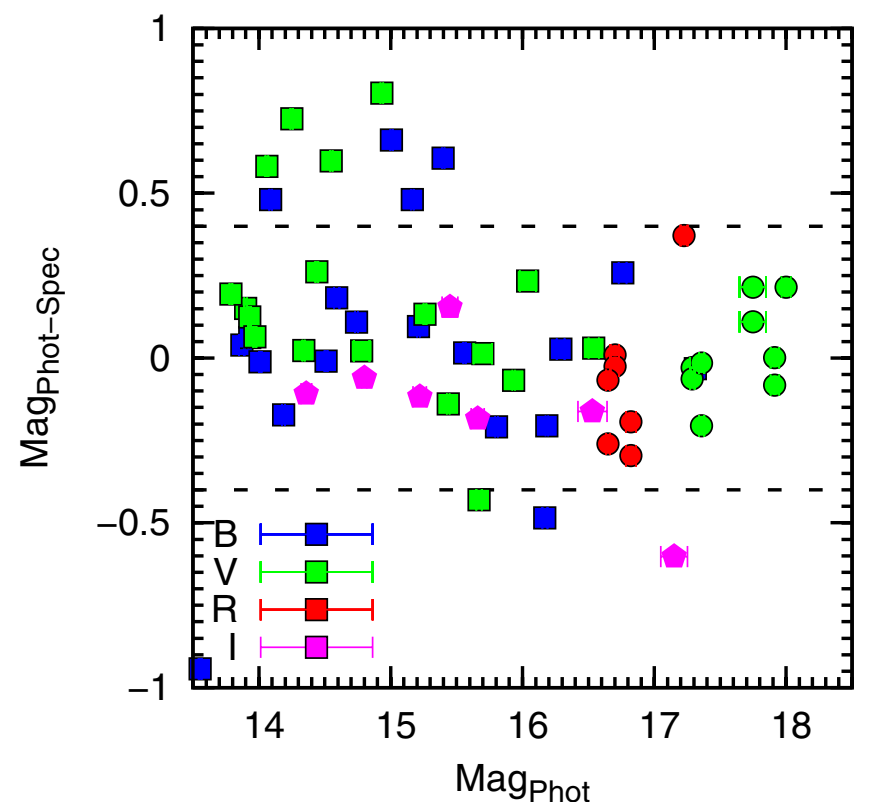

Fig. 12. Synthetic magnitudes as measured from flux-calibrated spectra compared to the photometric magnitude at the same epoch for SN 2009ip (Fraser et al. 2013, Gr\#11 and Gr\#16) SN 2012fr; (Childress et al. 2013, and Conteras et al., in prep; Gr\#11 and Gr\#16) SN 2013ai (Fraser et al., in prep; Gr\#13). Mag Phot is the calibrated photometric magnitude and the $y$-axis is the difference between this and the synthetic photometry measured from the flux calibrated spectra. Colours indicate filters, square symbols are Gr\#11, pentagons are Gr\#16 and circles are Gr\#13.

the standard deviation formally as $15.2 \%$ in the file headers but do not attach significance to the decimal digit. In future data releases we plan to significantly improve on the flux calibration scatter and reduce both the failures and intrinsic scatter. Reviewing the monthly sensitivity curves and applying photometric calibrations from the $V$-band acquisition images are the two most promising routes. The $V$-band acquisition images would allow a constant offset to be applied in an automated way, but only if all sky catalogues were available. In the future, the combination of Pan-STARRS1 (Magnier et al. 2013) and SkyMapper (Keller et al. 2007) will supplement SDSS DR9 (Ahn et al. 2012) to provide this all-sky reference catalogue and would allow an adjustment to the flux to bring down the absolute flux error to probably a few per cent. PESSTO will pursue this type of calibration as far as the reference catalogues will allow in future data releases.

We carried out further checks to determine the relative flux calibration across the EFOSC2 spectra compared to photometric measurements. We employed the $B V$ photometry of Fraser et al. (2013) for SN 2009ip and determined synthetic photometry from the EFOSC2 Gr\#11spectra, with the results plotted in Fig. 13. The average offset of these 14 spectra gives $(B-V)_{\text {spec }}-(B-V)_{\text {phot }}=0.05 \pm 0.04 \mathrm{mag}$ (where the error is the standard deviation of the individual differences). For Gr\#13 spectra, we used VR photometry of SN 2013ai from Fraser at al. (in prep.) and again the results are shown in Fig. 13. The 10 spectra give an average of $(V-R)_{\text {spec }}-(V-R)_{\text {phot }}=-0.05 \pm 0.05 \mathrm{mag}$.

The comparison plots indicate that there may be a systematic trend. It could indicate that the spectra of brighter objects are $\sim 5 \%$ redder than the photometry would imply. Or that the Gr\#11 spectra and Gr\#13 spectra have systematic offsets of +0.05 mag and -0.05 mag in comparison to what the photometry would imply. However this is not completely clear, since the
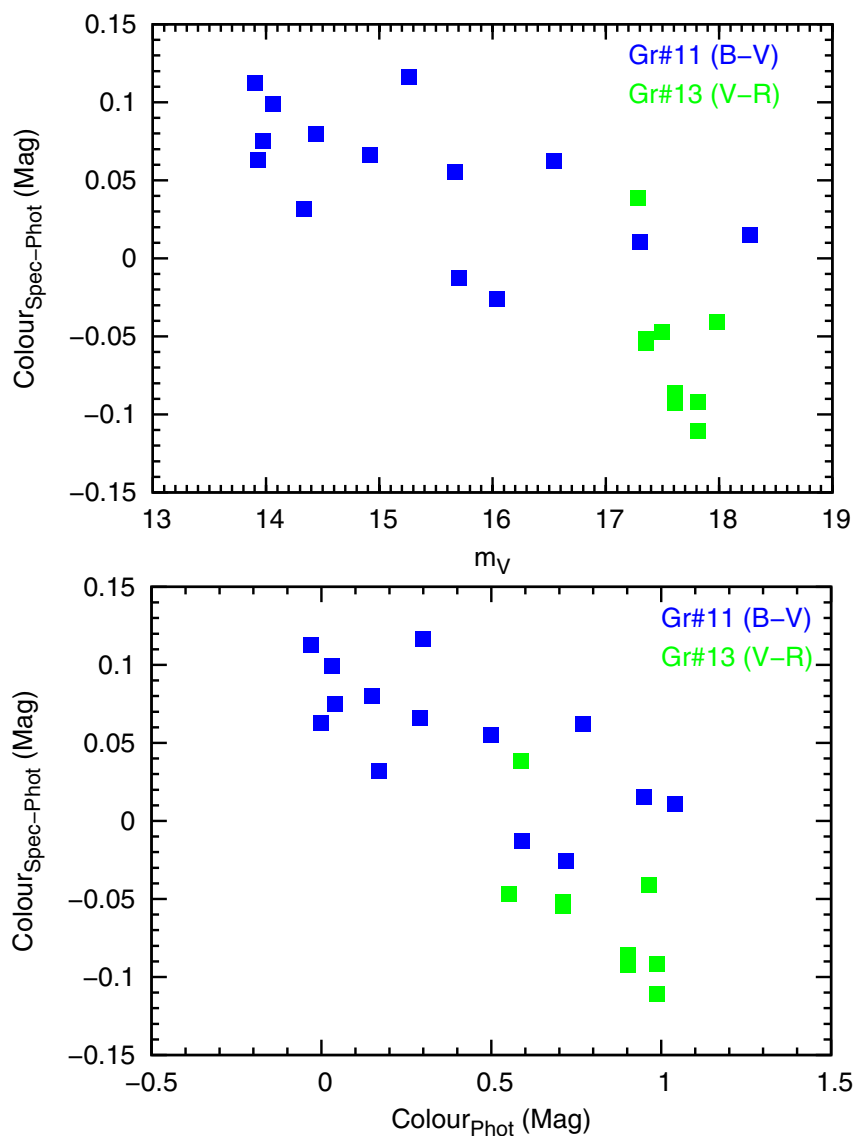

Fig. 13. A check on the relative flux calibration of the PESSTO spectra. The difference between the synthetic photometry colours of SN 2009ip (Gr\#11) and SN 2013ai (Gr\#13) and photometric measurements is plotted on the $y$-axis. The $x$-axis is simply the $V$-band photometric magnitude on the left panel and photometric colour (either $B-V$ or $V-R$ ) on the right.

average uncertainties in the photometric points are \pm 0.04 mag for SN 2009ip and \pm 0.05 mag for SN 2013ai (Fraser et al. 2013, Fraser et al., in prep.). We should also note that the SN 2013ai photometry comes from SMARTS $1.3 \mathrm{~m}$ telescope and KPNO filters. These are different to Johnson and Bessell filters and one should ideally do an S-correction on the photometry for consistent comparison. These trends will be probed further when we have more calibrated photometry and can investigate the trends with better statistics. From this preliminary investigation, we suggest that the relative flux calibration in the PESSTO spectra is accurate to around $5 \%$.

\subsection{Telluric absorption correction}

PESSTO does not specifically observe telluric standards for EFOSC2 such as fast rotating, smooth continuum stars. Instead, the data reduction pipeline uses a model of the atmospheric absorption to correct for the $\mathrm{H}_{2} \mathrm{O}$ and $\mathrm{O}_{2}$ absorption. The model was computed by F. Patat using the Line By Line Radiative Transfer Model (LBLRTM; Clough et al. 2005). Details on the model and the parameters used can be found in (Patat et al. 2011). This is carried out for all grism set-ups. The intensities of $\mathrm{H}_{2} \mathrm{O}$ and $\mathrm{O}_{2}$ absorptions in the atmospheric absorption model are first Gaussian smoothed to the nominal resolution of each instrumental set-up, and then rebinned to the appropriate pixel dispersion. The pipeline then scales the model spectrum so 
that the intensities of $\mathrm{H}_{2} \mathrm{O}$ and $\mathrm{O}_{2}$ absorptions match those observed in the spectrophotometric standards, hence creating multiple model telluric spectra per night. Each science spectrum is then corrected for telluric absorption, by dividing it by the smoothed, rebinned, and scaled absorption model which is most closely matched in time i.e. closest match between the standard star observation time and the science observation time.

\section{PESSTO EFOSC2 imaging observations and calibrations}

EFOSC2 is used in imaging mode for PESSTO to provide supporting photometry for some targets. Much of the photometric lightcurve data is provided by PESSTO scientists through their access to other facilities such as the SMARTS $1.3 \mathrm{~m}$ (DePoy et al. 2003), Liverpool Telescope (Steele et al. 2004) the LCOGT facilities (Boroson et al. 2014) the SWOPE $1 \mathrm{~m}$, (Perez et al. 2012) Asiago Telescopes (Tomasella et al. 2014) and PROMPT (Reichart et al. 2005). However EFOSC2 is also used for supporting data, particularly when the targets are fainter than around $19.5^{\mathrm{m}}$. The detector set-up is exactly the same as for the spectroscopic observations as described above in Sect. 3.1, and during each PESSTO night the filter wheel is loaded with the filters $U \# 640, B \# 639, V \# 641, R \# 642, g \# 782, r \# 784$, i\#705, z\#623. These filters have typically been employed in $U B V R i$ or $U g r i z$ sequences depending on the science target. Additionally, an acquisition image is taken through a $V$-band filter before every spectroscopic exposure to identify the target and allow it to be placed on the slit. These are also processed in a similar manner to the photometric science frames. The data final products and access are described in Sect. 5.

\subsection{EFOSC2 imaging calibration frames and reduction}

As the EFOSC2 CCD is read out in the same mode for both imaging and spectroscopy the CCD characteristics as discussed in Sect. 3.1 apply and the bias subtraction calibration is carried out as described in Sect. 3.2.1. The filters used for imaging are listed in Table 3 and their throughputs are illustrated in Fig. 14 (data taken from ESO database). Cosmic ray cleaning is generally applied to the full frame imaging data, as described in Sect. 3.2.3, and a header keyword is set to alert the user that this process has been run (see Appendix A.2).

Twilight sky flatfields for imaging are typically taken once per sub-run of $3-4 \mathrm{~N}$ in all of the eight filters (or as many as weather will allow). A master flat is created and used as close as possible to the science, or acquisition frames. The master flat and bias frames used for any particular frame can be found listed by in header keywords. The naming nomenclature is similar to that for the spectroscopic calibration frames but without the grism and slit names.

ZEROCOR = 'bias_20130402_56463.fits'

FLATCOR $=$ 'flat_20130401_R642_56463.fits'

In constructing these, the individual flats are checked and those with a high number of visible stars are rejected and not included in the masterflat. The masterflats commonly show a feature of apparent "dots" in a straight line (along X) in the central pixel area of [200:700,530:590] in filters Vgrz (it is also faintly visible in $B$ ). These are common, but transitory, and it is not clear if they are illumination ghosts and hence not present in the science frames. However, the counts level of these patterns differ by only $1 \%$ from the average level of the masterflat and as a consequence we assume they do not impinge on science frame calibrations.
Table 3. PESSTO photometric standard fields.

\begin{tabular}{lccc}
\hline \hline Standard field & RA (J2000) & Dec (J2000) & Filters \\
\hline T-Phe & 003014.00 & -463200.00 & UBVRi \\
PG0231+051 & 023341.00 & 051843.00 & UBVRgriz \\
RU149 & 072415.40 & -003207.00 & UBVRgriz \\
RU152 & 072956.00 & -020539.00 & UBVRgriz \\
PG1047+003 & 105005.65 & -000111.30 & UBVRgriz \\
PG1323-085 & 132549.00 & -085024.00 & UBVRgriz \\
PG1633+099 & 163534.00 & 094617.00 & UBVRi \\
PG1657+078 & 165933.00 & 074219.00 & UBVRi \\
MarkA & 204358.00 & -104711.00 & UBVRi \\
PG2336+004 & 233843.00 & 004255.00 & UBVRgriz \\
\hline
\end{tabular}

Imaging fringe frames are constructed for the $i$-band filter from a collection of NTT $i$-band images taken between Jan. 2010 and Apr. 2012.

PESSTO uses a set of 10 photometric standard fields, six of these 10 fields are photometric standards in both the Landolt and Sloan Digital Sky Survey (SDSS) systems (listed in Table 3). If the night appears to be photometric to the observers then a photometric standard field is observed three times. As with the spectroscopic standards, at least two of these should be different fields. During nights which are clearly non-photometric, PESSTO does not tend to take standard field calibrations. The PESSTO observers record their night reports on the PESSTO public web pages ${ }^{11}$ and record their judgment of whether the night is photometric or not. This page is publicly available and is a useful guide when interpreting the flux calibrations and validity of zeropoints in the FITS headers of the imaging files. The information is also recorded in the Table B.1.

The PESSTO pipeline is constructed to rapidly determine zero points $(\mathrm{ZP})$. Instrumental magnitudes are calculated for standard stars using DAOPHOT aperture photometry routines with an aperture set to 3 times the measured FWHM in the image, which are then compared to catalogue magnitudes. We carried out this ZP calculation for all available EFOSC2 imaging of the PESSTO standard fields for period stretching back 3 years from April 2013 (along with a few points from observations of the PG2213-006 standard field which is not a nominated PESSTO field). Many of these data come from the Benetti et al. large programme (ESO 184.D-1140) and we built upon the choice of standards and experience of that. The ZP trends are shown for each band in Fig. 15 and in constructing this plot we rejected any night which had an outlying ZP more than 0.5 mag away from the average of the $5 \mathrm{ZPs}$ closest in time as these were almost certainly non-photometric nights. We then rejected measurements when the ZPs were greater than $1 \sigma$ from their neighbours within \pm 2 adjacent days. The resultant measurements are likely to be from photometric nights which is illustrated by the low scatter and long term trends in Fig. 15. The cyclical long term trends are probably due to the ZPs being maximised immediately after re-aluminisation of the primary mirror (annually) and slow degradation afterwards. An average of ZPs and colour terms from Aug. 2012 until April 2013 is reported in Tab. 4. For PESSTO standard fields taken during PESSTO time the image products in the archive have zeropoints calculated directly with the Landolt or SDSS magnitudes of the stars in the field, and this is recorded in the header. The pipeline also provides ZPs for science frames if the fields are in the SDSS DR7 footprint and uses reference stars from that catalogue to set the ZPs. If

\footnotetext{
11 http://wiki.pessto.org/pessto-wiki/home/ night-reports
} 


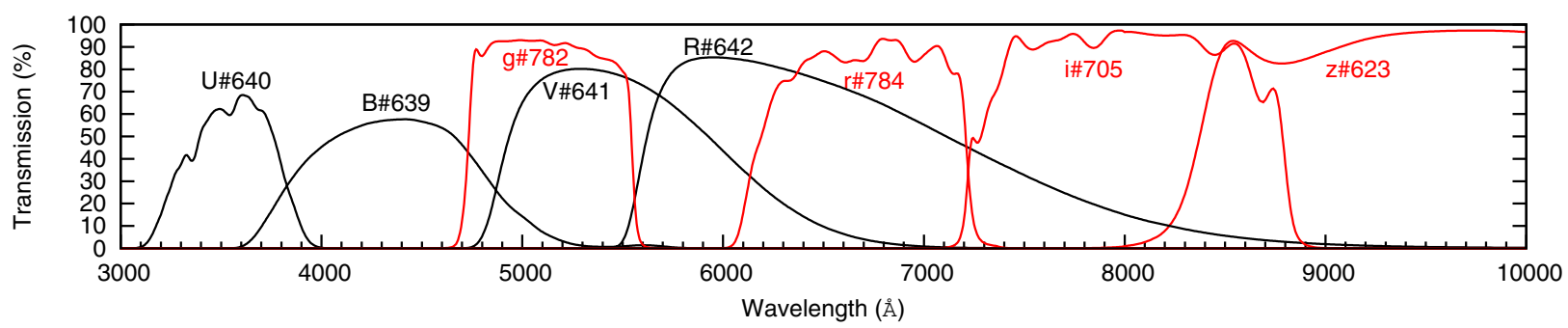

Fig. 14. Filter functions taken from the ESO database. The black lines are for the Landolt filters, while the red lines are for the Gunn filters. The $z$ filter does not have a cut-off in the red, but is instead limited by the quantum efficiency of the CCD, which drops to $10 \%$ at $1 \mu \mathrm{m}$.

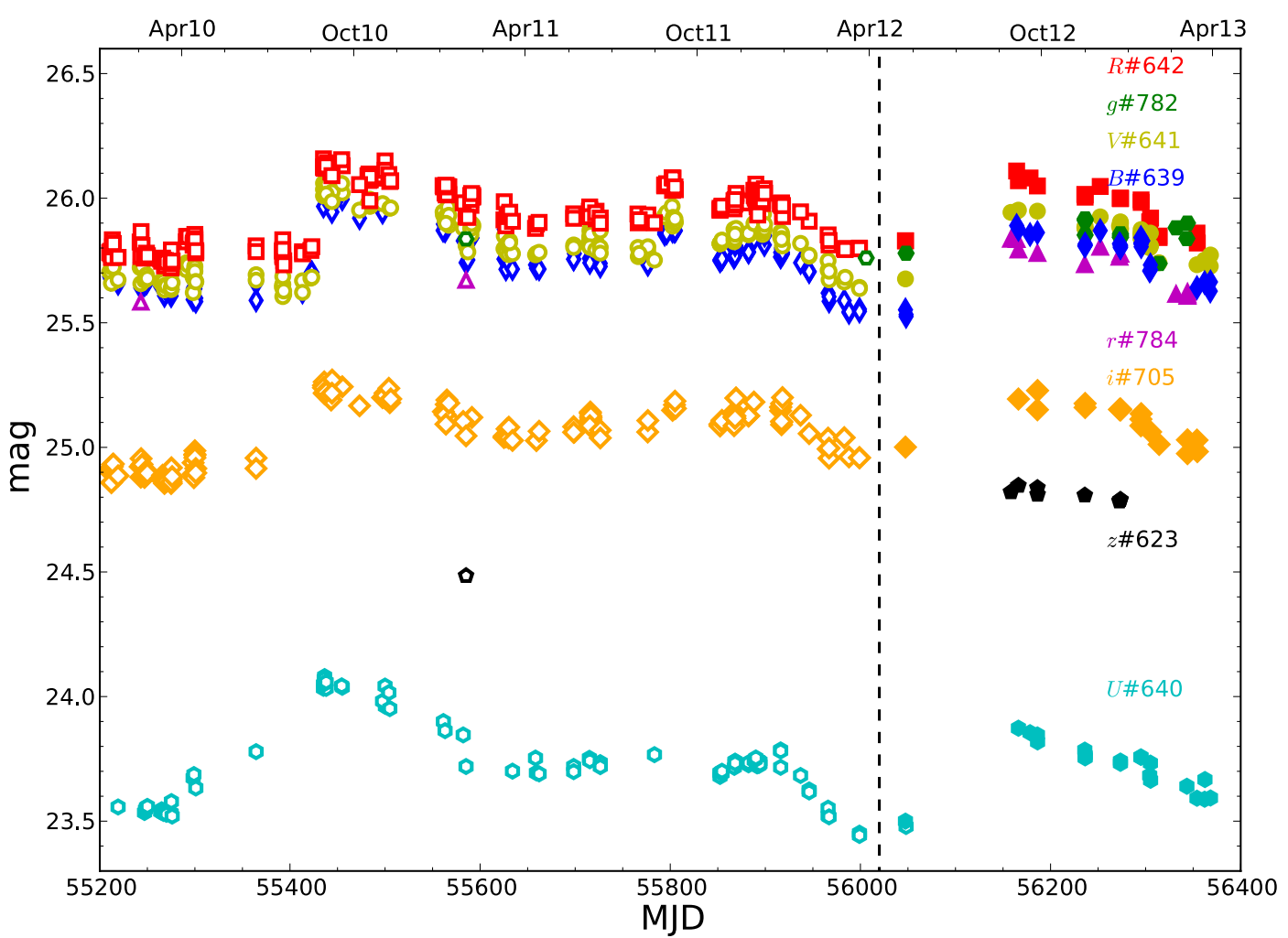

Fig. 15. Evolution of NTT zero points between 2010 and 2013. PESSTO data are shown by filled symbols, while the open symbols refer to archival data. The vertical dashed line indicates the first PESSTO night. ZPs were evaluated from observations of PESSTO standard fields using the PESSTO pipeline.

the science frame is observed with filters $g \# 642, r \# 784$ or $z \# 623$ the ZPs are provided in the SDSS AB system. If the science frame is observed with filters $U \# 640, B \# 639, V \# 641, R \# 642$, or $i \# 705$ the magnitudes of the stars in the SDSS DR7 catalogue are converted to the Landolt system using the equations from Jester et al. (2005) and ZPs are provided in Landolt system. For these cases when the field is in the DR7 footprint, the ZPs are calculated as follows. Instrumental magnitudes are calculated for reference stars matched to DR7 stars and are reported for an airmass $=0$ using the extinction coefficient reported in Table 4 . The $\mathrm{ZP}$ is computed as the mean of all ZPs obtained for all the stars that have catalogue matches. The PESSTO pipeline adds the following keywords which describe the data product, the measurements of which are described in full in Appendix A.7.

PSF_FWHM $=1.32371928 /$ Spatial resolution (arcsec)

$\begin{array}{lr}\text { PSF_FWHM }= & 1.32371928 / \text { Spatial resolution }(\operatorname{arcsec}) \\ \text { ELLIPTIC }= & 0.131 / \text { Average ellipticity of point sources }\end{array}$

$\begin{array}{ll}\text { ELLIPTIC } & 0.131 / \text { Average ellipticity of point } \\ \text { PHOTZP } & =25.98 / \mathrm{MAG}=-2.5 * \log (\text { data })+\text { PHOTZP }\end{array}$

$\begin{array}{lr}\text { PHOTZP }= & 25.98 / \mathrm{MAG}=-2.5^{*} \log (\text { data } \\ \text { PHOTZPER }= & 999 / \text { error in PHOTZP }\end{array}$

$\begin{aligned} \text { PHOTZPER } & =\text { 999 / error in PHOTZP } \\ \text { FLUXCAL } & =\text { 'ABSOLUTE' }\end{aligned}$

FLUXCAL = 'ABSOLUTE', / Certifies the validity of PHOTZ
PHOTSYS = 'VEGA

PHOTSYS $=$ 'VEGA,$/$ Photometric system VEGA or AB
ABMAGSAT $=13.34036 /$ Saturation limit for point sources (AB mags)

ABMAGSAT $=13.34036 /$ Saturation limit for point sources (AB mags)
ABMAGLIM $=19.86138 / 5$-sigma limiting AB magnitude for point sources
For images which do not fall in the SDSS DR7 footprint, we generally do not have reference stars in the EFOSC2 $4.1 \times 4.1$ arcmin field. Hence we adopt and report the average PHOTOZP which we have measured and recorded in Table 4. If the night was photometric, then the error in the PHOTZP is recorded as the error reported in Table 4 (PHOTZPER) allowing the user to use the PHOTOZP with some degree of confidence within the observed spread of the average measurement. If the night was not photometric, or we are unsure, then PHOTZPER is always set to 999 . The record of photometric and non-photometric nights are recorded by the observers on the PESSTO wiki ${ }^{13}$ and in Appendix B. In this way the keyword FLUXCAL is always set to ABSOLUTE, but users should be cautious of the validity.

Science users can then employ the ZPs to calibrate photometry of stars in the field using the following equation (and with the calibration caveats described above):

$$
\begin{aligned}
\text { MAG }= & -2.5 \times \log _{10}\left(\frac{\text { COUNTS }_{\mathrm{ADU}}}{\text { TEXPTIME }}\right) \\
& +\left(\text { AIRMASS } \times K_{\text {filter }}\right)+\text { PHOTZP }
\end{aligned}
$$


A\&A 579, A40 (2015)

Table 4. Average values of zero points and colour terms from the period Aug. 2012 until April 2013, as shown in Fig. 15.

\begin{tabular}{lccrc}
\hline \hline Filter & Zero point & Extinction coefficient & Colour term & \\
\hline$U \# 640$ & $23.655 \pm 0.090$ & $0.46 \pm 0.09$ & $0.096 \pm 0.030$ & $(U-B)$ \\
$B \# 639$ & $25.755 \pm 0.078$ & $0.27 \pm 0.05$ & $0.040 \pm 0.020$ & $(B-V)$ \\
$V \# 641$ & $25.830 \pm 0.075$ & $0.12 \pm 0.04$ & $0.034 \pm 0.018$ & $(B-V)$ \\
& & & $0.048 \pm 0.045$ & $(V-R)$ \\
$R \# 642$ & $25.967 \pm 0.079$ & $0.09 \pm 0.05$ & $0.031 \pm 0.042$ & $(V-R)$ \\
& & & $0.025 \pm 0.029$ & $(R-I)$ \\
$g \# 782$ & $25.897 \pm 0.085$ & $0.20 \pm 0.02$ & $0.073 \pm 0.031$ & $(g-r)$ \\
$r \# 784$ & $25.673 \pm 0.082$ & $0.09 \pm 0.01$ & $0.044 \pm 0.033$ & $(g-r)$ \\
& & & $0.056 \pm 0.045$ & $(r-i)$ \\
$i \# 705$ & $25.112 \pm 0.081$ & $0.02 \pm 0.01$ & $-0.014 \pm 0.015$ & $(r-i)$ \\
$z \# 623$ & $24.777 \pm 0.081$ & $0.03 \pm 0.01$ & $0.126 \pm 0.042$ & $(i-z)$ \\
\hline
\end{tabular}

Notes. The errors are standard deviations of the sample.

where $C O U N T S_{\mathrm{ADU}}$ is the measured signal in $\mathrm{ADU}$ and $K_{\mathrm{filter}}$ is the average extinction coefficient listed in for each filter in Table 4. The other terms are as defined in the FITS headers. Colour terms are not included, but are listed in Table 4 for reference.

The astrometric calibration was derived using the USNO B1 and 2MASS reference catalogues, and a distortion model described by a second-order polynomial. The astrometry task within the PESSTO pipeline employs the IMAGES package which is part of PYRAF. The pipeline makes an initial estimate for the astrometric solution of the field and iterates at least three times to reach a confidence level $<2$ arcsec in both $\alpha$ and $\delta$, otherwise it will record a failure to match catalogued stars. This is recorded in the FITS header with a value of 9999 for the keyword ASTROMET. A typical scatter of 0.4-0.5 arcsec was found for the science frames with around 15 stars usually recognised by the catalogue in the EFOSC 2 frame. This typically improves to an rms $\sim 0.2-0.3$ with $\gtrsim 30$ stars. For standard star fields we typically find a scatter of 0.2 arcsec, although the Landolt fields PG0231 and PG2336 usually produced a higher rms of $\sim 0.3-0.5$. The information on the rms of $\alpha$ and $\delta$ and the number of stars used for the calibration are given by the header keyword ASTROMET. Details for the other astrometric keywords are provided in Appendix A.8.

\section{PESSTO EFOSC2 data products}

\subsection{EFOSC2 Fast Reduced Spectra}

Since the start of PESSTO survey operations in April 2012, we have been releasing reduced spectra of all transient targets which have been classified by PESSTO and announced via the Astronomer's Telegram system within $24 \mathrm{~h}$ of the data being taken. These spectra are referred to as PESSTO "Fast Reduced Spectra", they are produced instantly at the telescope by the PESSTO observers. A support team in Europe or Chile is always on duty to either re-reduce these, or check them before they are made available publicly through WISeREP ${ }^{12}$ (Yaron \& Gal-Yam 2012). Only EFOSC2 spectra are produced as "Fast reduced spectra" (EFOSC2 FRS) as we do not use SOFI for classifications. These FRS are not ESO Phase 3 compliant and are an intermediate product to assist the survey and the public with good, but not final, data products. They are not sent to the ESO archive (although the raw data are immediately available in the ESO archive), and are not as carefully calibrated as the SSDR1

\footnotetext{
${ }^{12}$ http://www. weizmann.ac.il/astrophysics/wiserep/
}

spectra. They are only ever made available through WISeREP. The major differences are that flat fielding, bias image subtraction, fringe correction, and telluric absorption correction are not applied and a library sensitivity curve is employed for flux calibration. This section describes the data product, but we emphasise that these FRS data are now replaced with the fully reduced SSDR1 spectra in WISeREP and the ESO archive only includes the full reduced spectra.

The PESSTO pipeline has a module to produce the FRS from the three fixed EFOSC2 set-ups. These are not flat-fielded and the bias level is effectively removed during the sky-subtraction process. Wavelength calibration is achieved by applying a dispersion solution from an archive arc frame, which is not the one taken during the previous afternoon's calibrations. However an archival reference night sky spectrum is cross-correlated with the object frame's sky spectrum and a linear offset is applied to bring the dispersion solution into agreement with the observed night sky. As noted above in Sect. 3.2.4, the EFOSC2 dispersion solution is stable over long periods and we find typical observed shifts are 10-30 then results in residuals between the observed sky spectrum and the reference archive spectrum of less than a pixel. An average sensitivity curve for each of the EFOSC2 grisms is applied and the PESSTO pipeline hence produces wavelength calibrated, and flux calibrated 1D and 2D images. There is no correction applied for the telluric absorption lines. Bias and flatfields from the night, or indeed the observing run are not employed in the FRS. The PESSTO pipeline then allows the user to interactively select the object for extraction and set the background regions for background subtraction within the familiar IRAF apall package, and then carry out tracing and extraction. The extracted spectra are wavelength calibrated and then flux calibrated with an archive sensitivity function, after correcting for La Silla atmospheric extinction (Stritzinger et al. 2005). Cosmic ray rejection is generally turned on for these FRS, as described in Sect. 3.2.3.

This procedure is carried out by the observers at the NTT, or the backup data reduction and analysis team that PESSTO organises each month. The backup team can access the raw data at the end of Chilean night, and complete these reductions. Classifications are then made using one (or a combination) of the SN classification tools SNID (Blondin \& Tonry 2007), GELATO (Harutyunyan et al. 2008), or SuperFIT (Howell et al. 2005). These three codes each have different approaches and underlying assumptions. Their application and performance was discussed recently in Tomasella et al. (2014) in the context of the Asiago Supernova classification program. The codes each have a different set of database spectra for use in the classification 
algorithm. They can provide different answers for "best classification" depending on the signal-to-noise of the spectra and input information such as redshift and reddening. SNID is probably the most efficient algorithm, if the redshift of the SN or host galaxy is not known, and it also has a library of non-supernova spectra such as M-stars, AGN and luminous blue variable stars. Conversely, SNID assumes that the input spectrum is purely flux from the supernova, while SuperFIT can adjust the fit to include host galaxy contamination. GELATO has quite an extended library of spectra which is regularly updated and has a web-based interface which has recently undergone some improvements including a variable extinction option and smoothing algorithms (as described in Tomasella et al. 2014). As one might expect, for spectra with reasonable signal-to-noise $(S / N \gtrsim 15$, as is typical in PESSTO), the results from all three are in reasonable agreement. If the continua are featureless, or have shallow absorption or weak emission then one needs to be careful, irrespective of the code used. In PESSTO, if the classification is ambiguous then more than one of the classifiers is always used and the best estimate is provided at the point of classification. These classifications, based on the FRS spectra, are posted to the Astronomer's Telegram website, or occasionally (mostly in the case of amateur and TOCP discoveries) to the IAU Central Bureau. These spectra are uploaded to WISeREP and are immediately publicly available. The PESSTO target turn around time for this process is $24 \mathrm{~h}$ after the end of the Chilean night, and to date we have managed this on every night, save a very small number of exceptions. Some targets have uncertain classifications due to noisy spectra or contamination by host galaxy light. If a reasonable guess at classification cannot be made, the spectra are anyway made publicly available in WISeREP. In many cases a second attempt is made, particularly for those targets that have reasonable signal and defy standard classification. The most common type that we find are objects with blue featureless continua, which are often classified when more spectra are taken.

A comparison of the FRS and the SSDR1 spectra for a PESSTO classification target (SN 2012fx; also known as PSN J02554120-2725276) is shown in Fig. 5. Aside from a uniform scaling in flux, the overall appearance of the spectrum in the two reductions is very similar. In the rapid reduction, the uncorrected telluric $B$-band at $\sim 6870 \AA$ is apparent (the stronger $A$-band is lost in the deep OI absorption seen in the SN spectrum), while the rapid reduced spectrum also appears somewhat noisier in the red. In both cases, SNID finds the same best fitting template $(\mathrm{SN} 1991 \mathrm{bg}, z=0.018$, age $+1.9 \mathrm{~d}$ ), giving us confidence that the rapid reduced spectra are adequate for classification purposes.

\subsection{EFOSC2 final data product : SSDR1}

The Spectroscopic Survey data release 1 (SSDR1) is now available through the ESO archive system. This serves the survey data products which have been through the final data reduction process via the PESSTO pipeline. The data processing steps that have been applied are summarised below.

1. Bias subtraction: applied as described in Sect.3.2.1.

2. Flat fielding: for Gr\#11 and Gr\#13 flat fields from afternoon dome flats are applied. For Gr\#16, contemporaneous flat fields taken at the same instrument and telescope position as the science frames are applied (see Sect. 3.2.2). For spectrophotometric standards, daytime dome flats are used for all grisms.
3. Wavelength calibration: the 2D images are calibrated using arc frames as described in Sect. 3.2.4.

4. Cosmic Ray cleaning: the 2D images are cleaned of cosmic rays using the Laplacian cosmic ray rejection algorithm as discussed in Sect. 3.2.3.

5. Object extraction and background subtraction: the PESSTO pipeline implements the standard IRAF task apall to extract the target and apply background subtraction. This has been run in interactive mode by the data reduction team at Queen's University during the preparation of the SSDR1 data. This process is the most manual and user intensive in any spectroscopic data reduction process and if the transient object is on, or close to, a bright host galaxy then the choice of the background to subtract can be subjective. In all cases the PESSTO data reduction process has attempted to achieve a clean background subtraction to provide a target spectrum which is as uncontaminated as possible. The SSDR 1 also releases the fully calibrated (wavelength and flux) 2D frames as associated products for each $1 \mathrm{D}$ spectrum, so that a user can go back to this data product and simply re-extract with apertures and background regions of their choosing. This will provide wavelength and flux calibrated spectra, without having to go through all the reduction steps manually. The apall task has been run in pyraf with the multispec output format with variance weighting implemented. Hence each science spectrum also has an associated error spectrum and sky background spectrum which are the standard outputs from this process. The error spectrum produced by apall is the standard deviation of the variance weighted science spectrum.

6. Flux calibration: the 1D and 2D frames are flux calibrated as described in Sect.3.3.

7. Telluric absorption correction: this correction is applied as detailed in Sec.3.4. It is only applied to the 1D spectra, not the $2 \mathrm{D}$ calibrated images released as associated files.

The final step in both the EFOSC2 and SOFI spectral data reduction processes is to convert the one-dimensional flux calibrated spectrum images into binary FITS table format as the standard SSDR1 data products. These conform to the ESO Science Data Products Standard (Retzlaff et al. 2013), referred to as the spectrum binary table format. The binary table FITS file consists of one primary header (there is no data in the primary HDU so NAXIS $=0$ ), and a single extension containing a header unit and a BINTABLE with NAXIS $=2$. Although the binary FITS table format supports storing multiple science spectra within a single FITS file, a unique FITS file is provided for each individual science spectrum. The actual spectral data is stored within the table as vector arrays in single cells. As a consequence, there is only one row in the BINTABLE, which is NAXIS2 $=1$.

Information associated with the science spectrum is also provided within the same binary table FITS file resulting in a table containing one row with four data cells. The first cell contains the wavelength array in angstroms. The other three cells contain the science spectrum flux array (extracted with variance weighting), its error array (the standard deviation produced during the extraction procedure) and finally the sky background flux array. Each flux array is in units of $\mathrm{erg} \mathrm{cm}^{-2} \mathrm{~s}^{-1} \AA^{-1}$. A list of software that can be used to read spectra in binary FITS table is given in Appendix C.

The science spectrum has a filename of the following form, object name, date of observation, grism, filter, slit width, MJD of data reduction date, a numeric counter (beginning at 1) to distinguish multiple exposures taken on the same night, and a suffix _sb to denote a spectrum in binary table format. 


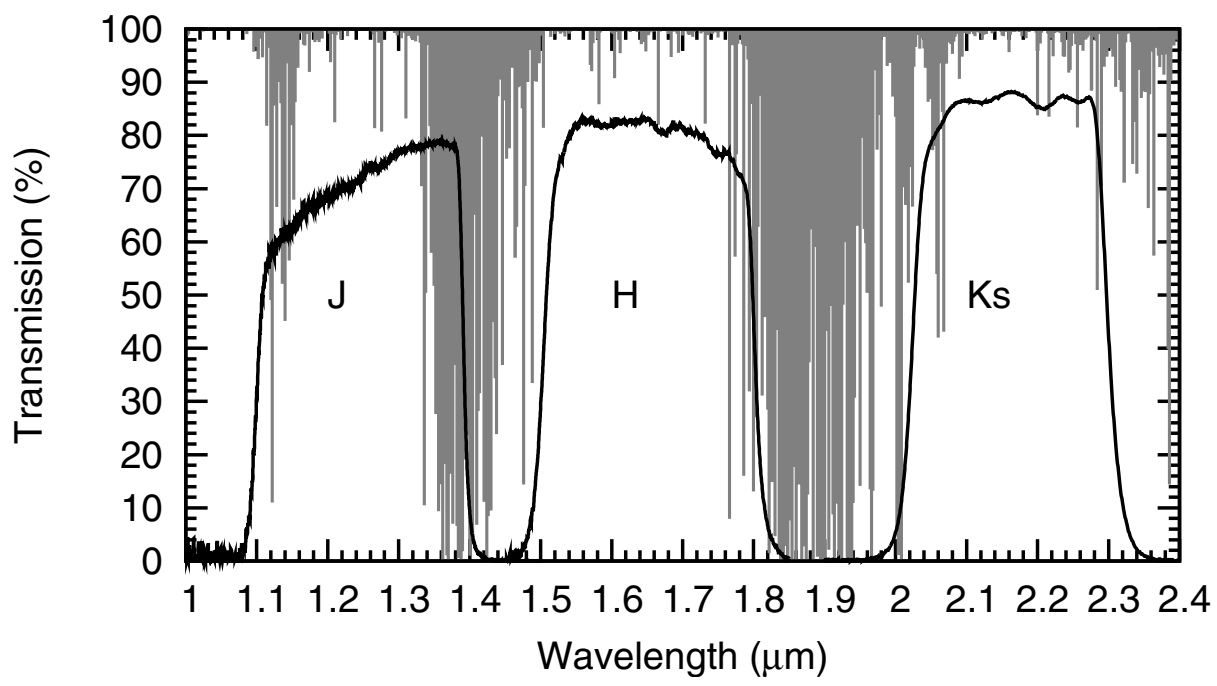

Fig. 16. Transmission curves for the JHKs filters used with SOFI. Also plotted in grey is the atmospheric absorption in the NIR (Lord 1992, courtesy of Gemini Observatory)

SN \,2013ak_20130412_Gr11_Free_slit1.0_56448_1_sb.fits

They can be identified as having the data product category keyword set as

PRODCATG $=$ SCIENCE.SPECTRUM $/$ Data product category

One should note that the ESO Science Archive Facility produces these files with a name which begins ADP and then appended with the date and time the file was created (as is standard ESO policy). The filename described here can always be retrieved from the FITS header with the keyword ORIGFILE.

The 2D spectrum images that can be used to re-extract the object as discussed above are released as associated ancillary data in SSDR1. They are associated with the science spectra through the following header keywords in the science spectra files. The file name is the same as for the 1D spectrum, but the suffix used is _si to denote an image.

ASSOC1 $=$ ANCILLARY.2DSPECTRUM / Category of associated file ASSON1 = SN2013ak_20130412_Gr11_Free_slit1.0_56448_1_si.fits /Name

These $2 \mathrm{D}$ files are wavelength and flux calibrated hence a user can re-extract a region of the data and have a calibrated spectrum immediately. Users should note the value for BUNIT in these frames means that the flux should be divided by $10^{20}$ to provide the result in $\mathrm{erg} \mathrm{cm}^{-2} \mathrm{~s}^{-1} \AA^{-1}$.

We are also releasing the reduced acquisition images and reduced multi-colour photometric follow-up frames. These are reduced as discussed in Sect. 4 and are currently available directly from the PESSTO website (www . pessto.org) and will soon be available in the ESO archive. As they don't all have reliable absolute photometric zeropoints (as described in Sect. 4.1) they will be available as associated ancillary data from ESO, rather than separate science data products. The acquisition images may be useful for improving on flux calibration in the future, if reference stars in the field can be accurately calibrated. Images have the following naming convention for acquisition and science frames respectively

acq_SN2012ec_20120907_V641_56462_1.fits

SN2011hs_20120422_R642_56462_2.fits

This naming scheme is similar to the spectral files: object name, observation date, filter (including ESO number), MJD of date of reduction and a numeric counter to distinguish multiple exposures from the same night. The acquisition images have the acq_prefix.

\section{PESSTO SOFI spectroscopic observations and calibrations}

The Son OF ISAAC (SOFI) ${ }^{13}$ is an infrared spectrograph and imaging camera which is mounted on the opposite nasmyth platform to EFOSC2 on the NTT (Nasmyth A focus) and has been installed there since 1997 (Moorwood et al. 1998). The instrument has a $1024 \times 1024$ Hawaii $\mathrm{HgCdTe}$ array with $18.5 \mu \mathrm{m}$ pixels. The array sensitivity and range of filters and grisms cover imaging and spectroscopy between $0.9-2.5 \mu \mathrm{m}$. PESSTO operates with the SOFI imaging and spectroscopy default modes which have pixel scales of $0{ }^{\prime} 29 \mathrm{pix}^{-1}$, and $0 ! 27 \mathrm{pix}^{-1}$, respectively due to the different objectives employed (Lidman, et al. 2012). The imaging mode provides a FOV of 4.9 arcmins. PESSTO uses the long slit spectroscopy mode with the two low-resolution grisms labelled "Blue" and "Red" and the wavelength coverage is listed in Table 5 and typically only takes spectra for targets which are in the magnitude range $14<H<17$. PESSTO does not use SOFI spectroscopy for any type of classification, only targets that are picked as PESSTO Key Science Targets are put forward for SOFI observations and only those bright enough to give reasonable signal-to-noise (typically $S / N \sim 20$ in the continuum) are spectroscopically observed. PESSTO also uses SOFI in imaging mode, using the filters $J H K_{\mathrm{s}}$, as shown in Fig. 16. The $K-$ short or $K_{\mathrm{s}}$ filter is different to standard $K$ and $K^{\prime}$ as it transmits between 2-2.3 $\mu \mathrm{m}$ hence avoiding the $1.9 \mu \mathrm{m}$ atmospheric absorption feature and cuts short of the increasing thermal background beyond $2.3 \mu \mathrm{m}$ (Lidman, et al. 2012). No other imaging filters are employed for PESSTO SOFI observations. The amount of SOFI NIR data available for any PESSTO science target depends critically on the brightness of the source and the science drivers. Hence, as originally planned in the survey proposal, SOFI observations make up around 20 per cent of the total PESSTO time.

\subsection{Detector characteristics}

The detector installed in SOFI is a Rockwell Scientific Hg:Cd:Te $1024 \times 1024$ Hawaii array with $18.5 \mu \mathrm{m}$ pixels and an average quantum efficiency of $65 \%$. It has a dark current of typically

\footnotetext{
13 http://www . eso.org/sci/facilities/lasilla/ instruments/sofi.html
} 
Table 5. PESSTO settings for SOFI spectroscopy.

\begin{tabular}{rlllllllll}
\hline \hline Grism & $\begin{array}{l}\text { Wavelength } \\
(\mu \mathrm{m})\end{array}$ & $\begin{array}{l}\text { Filter } \\
\text { (blocking) }\end{array}$ & $\begin{array}{l}n_{\text {pix }} \\
(\text { pixels })\end{array}$ & $\begin{array}{l}\text { Dispersion } \\
(\AA / \text { pixel })\end{array}$ & $\begin{array}{l}\text { Resolution } \\
(\AA)\end{array}$ & $\begin{array}{l}R \\
\lambda / \Delta \lambda\end{array}$ & $\begin{array}{l}V \text { resolution } \\
\mathrm{km} \mathrm{s}^{-1}\end{array}$ & Arclines & $\begin{array}{l}\text { rms } \\
\AA\end{array}$ \\
\hline Blue & $0.935-1.645$ & GBF & 1024 & 6.95 & 23 & 550 & 545 & $12-14$ & $0.1-0.2$ \\
Red & $1.497-2.536$ & GRF & 1024 & 10.2 & 33 & 611 & 490 & $7-8$ & $0.2-0.5$ \\
\hline
\end{tabular}

Notes. The order blocking filters used are $0.925 \mu \mathrm{m}$ (GBF) and $1.424 \mu \mathrm{m}$ (GRF) "cut-on" filters. A 1". slit projects to 3.4 pixels FWHM, measured from arc lines and the resolution $R$ is given at the midpoint of the spectral ranges, as is the velocity resolution. The column headed Arclines indicates the number of lines used. The rms is the typical residual for the wavelength calibration solution.

around $20 \mathrm{e}^{-} \mathrm{h}^{-1}$ per pixel, and a documented readout noise of approximately $12 \mathrm{e}^{-}$, both of which are negligible compared to background in PESSTO exposures. The gain of the array is $5.4 \mathrm{e}^{-} / \mathrm{ADU}$ and well depth around 170000 electrons (32000 ADU). The array non-linearity is reported to be less than $1.5 \%$ for a signal up to 10000 ADU (Lidman, et al. 2012), but the ESO instrument scientists recommend that exposures keep the background below 6000 ADU owing to the bias of the array, which has a complicated dependence on flux levels.

In imaging mode, we use DCR (double correlated read) mode which results in a readnoise of around $12 \mathrm{e}^{-}$. The short noise from the sky (or object if it is bright) dominates and readnoise is negligible for imaging. In spectroscopy mode, we always use the NDR (non-destructive read) mode with the settings NSAMP $=30$ and NSAMPPIX $=4$ (as described in the SOFI manual; Lidman, et al. 2012). This mode is recommended for spectroscopy and the array is read within each DIT a number of times (equal to NSAMP), and for each read-out the signal is sampled NSAMPIX times. This mode reduces the readnoise further than for DCR, with read noise values typically in the range $2-3 \mathrm{e}^{-}$.

\subsection{SOFI spectroscopic calibration data and reduction}

Similar to PESSTO observations and reductions for EFOSC2, we aim to homogenise the SOFI observations and calibrations and tie them directly to what is required in the data reduction pipeline. A standard set of PESSTO OBs for calibrations and science are available on the PESSTO wiki and the following sections describe how they are applied in the pipeline reduction process.

\subsubsection{Bias, dark and cross-talk correction}

Unlike for EFOSC2, we do not subtract bias (or dark) frames from SOFI images or spectra. The bias level seen in the each image is dependent on the incident flux level, and so it is not practical to correct this with daytime calibration data. Instead, any bias offset or structure is subtracted along with the sky background, as recommended in the SOFI handbook.

The SOFI detector suffers from cross talk, where a bright source on either of the two upper or lower quadrants of the detector will be accompanied by a "ghost" on the corresponding row on the opposite two quadrants. This ghost will affect the entire row of the detector, and has a fixed intensity relative to the opposite row. This cross-talk effect is corrected for within the pipeline by summing each row on the detector, scaling by a constant value, and subtracting from the opposite quadrants.

\subsubsection{Flat field calibration}

Spectroscopic flats are taken approximately once per month for SOFI; these consist of pairs of flats, taken first with an

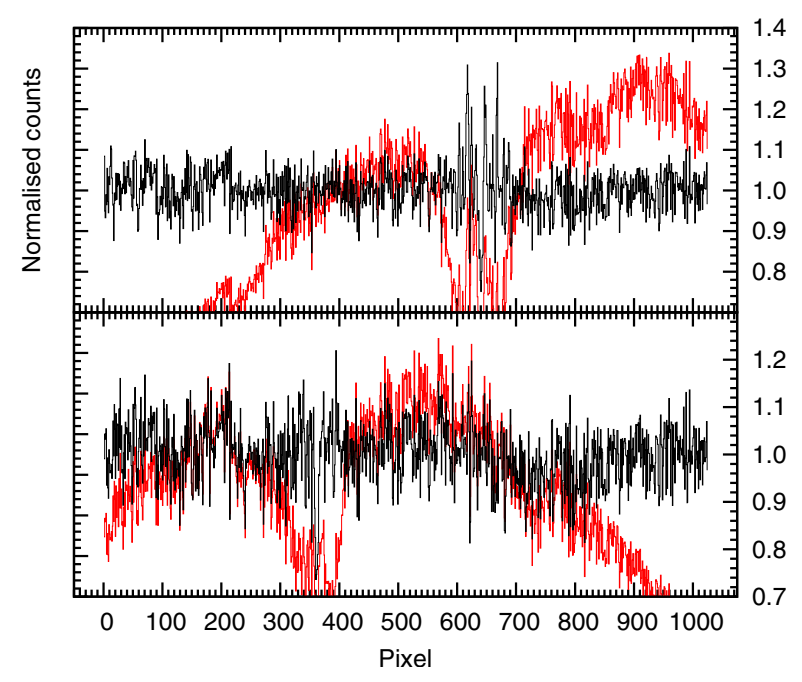

Fig. 17. Cut across SOFI flat fields along row 512. Blue grism (lower) and red grism (upper panel) are shown, with the normalised flat field shown in black, and the raw flat field (showing the $\mathrm{H}_{2} \mathrm{O}$ absorption) in red.

incandescent lamp illuminating the dome, and then with the dome un-illuminated. The lamp-off flats are subtracted from the lamp-on flats, to remove the thermal background of the system. These subtracted flat fields are then combined and normalised using a high-order (order 80 by default) spline fit; the normalised flat field is used to correct for the pixel to pixel variations in detector sensitivity in the science and standard star frames. Although atmospheric absorption features due to the light path between the dome lamp and the detector can be seen in these flats, the normalisation appears to remove them relatively well. The raw and normalised flat fields for both the blue and red grisms are shown in Fig. 17.

The amplitude of the variability in the flat field is $\sim 4 \%$ for the red grism and $\sim 6 \%$ for the blue grism. The pixel-to-pixel variation in Fig. 17 illustrates the real response of the detector, rather than being due to shot noise in the flats. We verify this in Fig. 18, where we compare a section of two normalised red grism flat fields taken $\sim 5$ months apart. Both flats show the same structure, demonstrating that the flat field is stable, and that the use of monthly calibrations is justified.

\subsubsection{Arc frames and wavelength calibrations}

As for the optical spectra, wavelength calibration is performed using spectra of a xenon arc lamp. To fit the dispersion solution of the arc spectra without any systematic residuals requires a fourth-order polynomial fit. As listed in Table 5, 7-8 lines were typically used for the fit in the red grism, and 12-14 lines in the blue grism, giving an rms error in the wavelength of around 


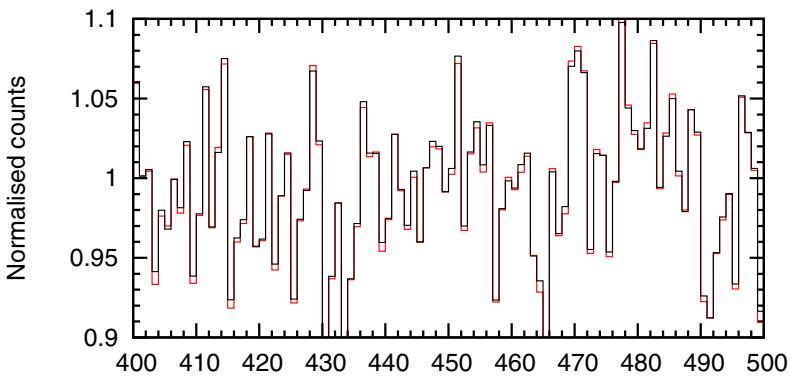

Fig. 18. Comparison of a cut along column 512 of the normalised flat for the red grism taken on 2013 March 3 (black) and the normalised flat taken on 2012 October 6 (red) between pixels 400 and 500. The two flats are essentially identical, indicating that the "noise" seen in Fig. 17 is in fact $\sim 5 \%$ pixel-to-pixel variation in the detector.

$0.2-0.5 \AA$. The dispersion solution found from the arc frames is then applied to the two dimensional spectra. The wavelength calibration is also checked against the sky lines. After the 2D science frame is wavelength calibrated, the frame is averaged along the spatial axes and cross-correlated with sky lines. A linear shift is applied to the wavelength calibration and recorded in the header keyword SHIFT. A more robust result is obtained if the regions of the spectrum containing strong telluric absorption is removed before the wavelength calibration check is performed. As with the EFOSC2 correction, the precision of the wavelength correction is limited to $1 \AA$, owing to the scale of the shifts in the library sky spectra employed. Hence this value of $1 \AA$, is again recorded as the systematic error in the wavelength calibration (SPEC_SYE, see Appendix A.3).

\subsubsection{Sky subtraction and spectral extraction}

A critical part of NIR observations is the bright sky background, which usually has higher flux levels than the target. The sky can vary on timescales of a few minutes, and so must be measured and subtracted at (or close to) the time of the science observations. To accomplish this, SOFI spectra for PESSTO are taken in an ABBA dither pattern. This pattern consists of taking a first $\left(\mathrm{A}_{1}\right)$ exposure at a position " $\mathrm{A}$ ", then moving the telescope so that the target is shifted along the slit of SOFI by $\sim 5-10$ " to position " $\mathrm{B}$ ". Two exposures are taken at " $\mathrm{B}$ " $\left(\mathrm{B}_{1}\right.$ and $\left.\mathrm{B}_{2}\right)$, before the telescope is offset back to "A" where a final exposure $\left(A_{2}\right)$ is taken. When reducing the data, the pipeline subtracts each pair of observations (i.e. $A_{1}-B_{1}, B_{1}-A_{1}, B_{2}-A_{2}, A_{2}-B_{2}$ ) to give individual bias- and sky-subtracted frames. Next, the PESSTO pipeline attempts to shift these sky-subtracted frames so that the trace of the target is at a constant pixel position, and combine the frames. If the target is relatively faint, and the spectral trace cannot be identified clearly in each frame, this routine in the pipeline will fail, and instead the user will be prompted to interactively align and combine the frames. Finally, the spectrum is optimally extracted in an interactive fashion.

The total on object exposure time of these combined frames is given in the header as TEXPTIME. This is simply a product of the following values, all found as header keywords : DIT (the detector integration time), NDIT (the number of DITs), NJITTER (the number of jitters at positions "A" and "B"), and NOFFSETS (the number of offset positions, which is always 2). Typically DIT is kept between 60-240 s.

\subsection{Telluric absorption correction}

The NIR region covered by SOFI contains multiple strong telluric absorptions, arising chiefly from water vapour and $\mathrm{CO}_{2}$, and their absorption strength is a function of both time and airmass. The most common technique for low-to-mediumresolution spectroscopy is to observe a star of known spectral type (a "telluric standard") immediately prior to or following the science spectrum, and at a similar airmass. The spectrum of the telluric standard is then divided by an appropriate template spectrum of the same spectral type, yielding an absorption spectrum for the telluric features. The absorption spectrum is then divided into the science spectrum to correct for the telluric absorption. As part of PESSTO, we observe either a Vega-like (spectral type A0V) or a Solar analogue (G2V) telluric standard for each SOFI spectrum. The PESSTO pipeline uses the closest (in time) observed telluric standard to each science or standard star spectrum.

\subsection{Spectrophotometric standards and flux calibration}

The process for correcting the spectrum for the telluric absorption also provides a means for flux calibration using the HIPPARcos $I$ or $V$ photometry of the solar analogues and Vega standards used. The flux of the observed telluric standard spectrum is scaled to match the tabulated photometry, with the assumption that the telluric standards have the same colour (temperature) as Vega or the Sun. When possible, a second step is performed to flux calibrate the spectra using a spectrophotometric standard. The spectrophotometric standard is reduced and corrected for telluric absorption using a telluric standard, with the same technique as used for the science targets. This corrected standard spectrum is then compared with its tabulated flux, and the science frame is then linearly scaled in flux to correct for any flux discrepancy. There are only a handful of spectrophotometric standard stars which have tabulated fluxes extending out as far as the $K$-band. We do observe these standards (listed in Table 2) as far as possible when SOFI spectra are taken, but nonetheless there are a significant number of nights where no flux standard was observed in the NIR. For these nights the spectra will still have an approximate flux calibration performed against the accompanying telluric standard. An example of a reduced and flux calibrated spectrum is shown in Fig. 19.

All SOFI spectra have the following keyword which denotes which telluric standard was used for both the telluric correction and the initial flux calibration.

SENSFUN = 'TSTD_Hip109796_20130417_GB_merge_56478_1_ex.fits' / tell stand frame

If one of the spectrophotometric flux standards from Table 2 has been used to additionally scale the flux then the keyword SENSPHOT is added to the header, with the spectrum used to apply the flux calibration. This file has the name of the standard clearly labelled. In this way, users can distinguish which method has been applied.

SENSPHOT='sens_GD71_20130417_GB_merge_56478_1_f.fits'/sens for flux cal

To check the flux calibration of SOFI spectra, we would ideally have a large number of targets with both well sampled NIR lightcurves and SOFI spectra. At this time, the NIR lightcurves for most of the PESSTO science targets are not complete and not calibrated reliably enough to allow a large scale comparison. We have used a well observed type Ia SN (SN 2012fr; Childress et al. 2013) to determine the accuracy and reliability of the SSDR 1 flux calibrations. Synthetic $J$-band photometry was performed 


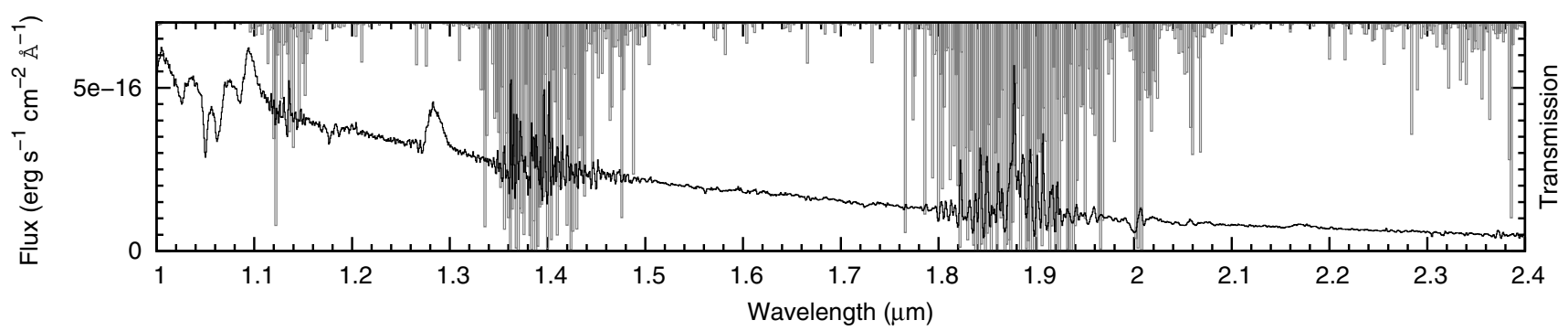

Fig. 19. Combined blue and red grism SOFI spectra of SN 2012ec taken on 2013 September 24. Overplotted in grey is the atmospheric transmission, showing the correspondence between regions of low transparency and poor $\mathrm{S} / \mathrm{N}$ in the spectrum.

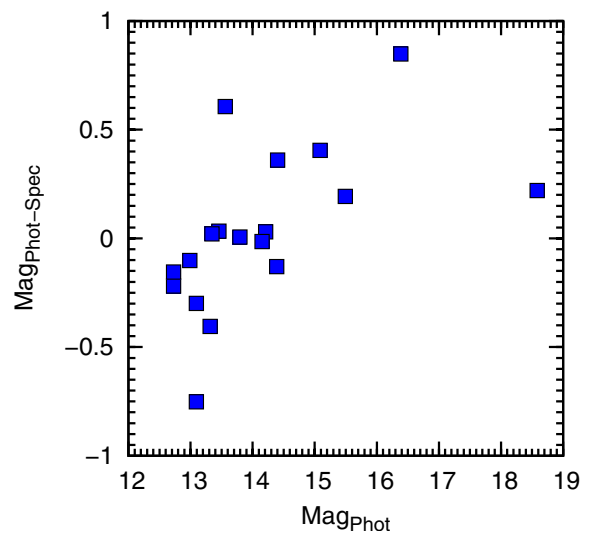

Fig. 20. Comparison of observed and synthetic $J H$ magnitudes for SN 2012fr. The standard deviation of the distribution is $34.7 \%$, which is a measure of the uncertainty of the absolute spectroscopic flux scale of the calibrated SOFI spectra.

on the blue grism spectra, and $H$-band photometry on the red grism spectra. The difference between the synthetic magnitudes and the $J H$ photometry from Conteras et al. (in prep.) is plotted in Fig. 20. Not surprisingly, a fairly large spread of magnitude offsets is seen, with the distribution having a mean of $0.04^{\mathrm{m}}$ and a standard deviation of $0.37^{\mathrm{m}}$. Although this is quite a significant scatter, it can be improved upon by users by employing the $J H K_{\mathrm{s}}$ imaging that is normally done when SOFI spectra are taken. Synthetic photometry will allow more accurate scaling of the absolute flux levels. This correction is not in SSDR1, but in future PESSTO data releases, the flux calibration of SOFI spectra will be cross checked against the $J H K_{\mathrm{s}}$ photometry of the target taken closest to the observations.

\subsection{SOFI imaging calibration frames and reduction}

SOFI imaging is carried out as default when spectroscopy is done, providing images with a 4.9 arcmin field of view $\left(0.29\right.$ pix $\left.^{-1}\right)$. The cross talk effect is first corrected as for the spectra and all images are then flat fielded using dome flats. Dome flats are taken using a screen on the interior of the telescope dome which can be illuminated with a halogen lamp. Pairs of flats are taken with the screen illuminated and un-illuminated; the latter are then subtracted from the former to account for dark current and thermal background. Multiple flats are combined, and then used to reduce the science data. Typically, dome flats are taken once per month with SOFI, although they appear stable over longer periods.

An illumination correction is also applied, to account for the difference between the illumination pattern of the dome flats and the actual illumination of the night sky. The illumination correction is determined by imaging a bright star at each position in a $4 \times 4$ grid on the detector. The intensity of the star is then measured at each position, and a two-dimensional polynomial is fitted. This polynomial is normalised to unity, so that it can be applied to the imaging data as a multiplicative correction. These on-sky calibrations are tested annually within PESSTO.

Sky-subtraction is the most important aspect of NIR imaging and reductions. For targets that are in relatively uncrowded fields, a dither pattern is employed where the telescope is moved to four offset positions on the sky, while keeping the target in the field of view ("on-source sky subtraction"). To determine the sky background, the four frames are then median combined without applying offsets, rejecting pixels from any individual image which are more than a certain threshold above the median. This initial sky image is subtracted from each individual frame in order to obtain initial sky-subtracted images. These frames are used to identify the positions of all sources and create a mask frame for each science image. For each set of four images, the frames are then median combined again without applying offsets and using the masks created previously to reject all sources and produce the final sky image. The final sky background image is then subtracted from each of the input frames. The skysubtracted images are then mosaiced together to create a single image using the SWARP package (Bertin et al. 2002).

For targets which are in a crowded field, or where there is extended diffuse emission (such as nearby galaxies), then onsource sky subtraction is not possible. In these cases, we alternate between observing the target, and observing an uncrowded off-source field around $\sim 5$ arcmin from the target. We typically observe four frames on source, then four frames off source, dithering in each case The off-source frames are then used to compute a sky frame in the same way as for the "on-source sky subtraction". The off-source sky frame is then subtracted from each of the on-source images of the target, which are then combined to create the final image. Since the field of view of SOFI is rather small (4.9 arcmin) the astrometry is not set for single images. Instead, SEXTRACTOR is run to detect sources in individual frames, and to check the nominal dither. The images are then mosaiced together using SWARP. Finally, an astrometric calibration is made, by cross correlating the sources detected by SEXTRACTOR with the 2MASS catalogue, in the same fashion as for the EFOSC2 frames. The instrumental aperture magnitudes of the sources in the field as measured by DAOPHOT are then compared to their catalogued 2MASS magnitudes to determine the photometric zeropoint, which is recorded in the header of the image as PHOTZP.

The definition of PHOTZP for SOFI is different to that of EFOSC2. Since the SOFI images all have astrometric and photometric solutions from 2MASS point source matching, it is possible to give a measured zeropoint for all images. The SOFI 
images are full science archive products and as such they obey the formal ESO definition of the zeropoint :

$\mathrm{MAG}=-2.5 \times \log _{10}\left(\mathrm{COUNTS}_{\mathrm{ADU}}\right)+\mathrm{PHOTZP}$

The extinction term is not used since the 2MASS calibration sources are in the same image, and the TEXPTIME term is incorporated into the PHOTZP value. The other photometric keywords are similar to EFOSC2 and are described in Appendix A.7.

\subsection{PESSTO SOFI data products: SSDR1}

PESSTO does not produce fast reduced spectra for SOFI, since the NIR is never used for classification. Hence only final reduced spectra and images are described here for SSDR1. The data products for SOFI are similar to those described in Sect. 5.2 for EFOSC2. The spectra are in binary table FITS format, with the same four data cells corresponding to the wavelength in angstroms, the weighted science spectrum and its error and the sky background flux array. Again, each flux array is in units of $\operatorname{erg~cm}{ }^{-2} \mathrm{~s}^{-1} \AA^{-1}$. (see Appendix $\mathrm{C}$ for a list of software that can read binary FITS table format). The SSDR1 FITS keywords described Appendix A.7 are again applicable here. A typical file name is

SN2009ip_20130417_GB_merge_56478_1_sb.fits

Where the object name is followed by the date observed, the grism (GB for the blue grism, or GR for the red grism), the word "merge" to note that that the individual exposures in the ABBA dither pattern have been co-added, the MJD date the file was created, a numeric value to distinguish multiple exposures on the same night and a suffix _sb to denote a spectrum in binary table format. As with EFOSC2, this science spectrum can be identified with the label :

PRODCATG $=$ SCIENCE.SPECTRUM $/$ Data product category

We also provide the 2D flux calibrated and wavelength calibrated file so that users can re-extract their object directly, as described with EFOSC2. The identification of the 2D images follow the same convention as for EFOSC2, with the suffix _si to denote a spectral image.

ASSOC1 = ANCILLARY.2DSPECTRUM / Category of associated file ASSON1 = SN2009ip_20130417_GB_merge_56478_1_si.fits / Name of associated file

We do not reduce and release the SOFI equivalent of the EFOSC2 acquisition images, but in nearly all cases where PESSTO takes a SOFI spectrum, imaging in $J H K_{\mathrm{s}}$ is also taken. These images are flux and astrometrically calibrated and released as science frames rather than associated files. They are labelled as follows where Ks labels the filter and the merge denotes that the dithers have been co-added.

SN2013am_20130417_Ks_merge_56475_1.fits

We also release the image weight map as described in Retzlaff et al. (2013). The definition in this document is the pixel-topixel variation of the statistical significance of the image array in terms of a number that is proportional to the inverse variance of the background, i.e. not including the Poisson noise of sources. This is labelled as

\section{Summary and data access}

This paper describes the processing and calibration of PESSTO data products that are served by ESO as the Spectroscopic Survey data release 1 (SSDR1). From this first year of science operations, a total of 909 reduced and calibrated spectra from EFOSC2 and SOFI for 298 distinct objects have been released along with 234 reduced and calibrated near-infrared SOFI images for 22 objects. These spectra and SOFI images are available from the ESO archive as Phase 3 compatible data products. In addition we make available the reduced and calibrated EFOSC2 images now, before they are fully ESO archive compliant. As of October 2014, PESSTO has classified around 570 transient objects and is carrying out follow-up campaigns on around 90 of these. All information is kept up to date on the PESSTO website to support this public survey and classification spectra are released on an ongoing basis via WISeREP (Yaron \& Gal-Yam 2012). As discussed in Sect. 1, one of the major goals of PESSTO is to study the extremes of the known transient populations and provide comprehensive datasets to study the physical mechanisms producing these objects. An illustrative diagram of the phase space of explosive and eruptive transients was first plotted by Kulkarni et al. (2007) to show the faint and relatively fast nature of transients in the gap between faint supernovae and novae. This was expanded by Kulkarni \& Kasliwal (2009) and Kasliwal et al. (2010) to higher luminosities and faster declining objects. As an illustration of PESSTO's science goals, Fig. 21 shows this Kulkarni \& Kasliwal diagram updated with objects that PESSTO has classified and is following. The data for these will be released in future public releases via the ESO archive, and this shows the extremes of the transient population that we are now covering extensively.

We have highlighted the difficulty in homogenising the flux calibration of small imaging fields in a public survey, and providing absolute spectroscopic flux calibration to below $10 \%$ across many nights which have variable seeing and transparency. However methods to improve these for future data releases have been identified and neither of these greatly affects the science of transient objects that can be done with PESSTO. Science users have the ability to adjust the flux measurements since the data releases contain enough information that improvements to the calibrations can be tailored for specific objects, with additional manual steps in calibrating. For example, as all EFOSC2 spectra have an acquisition image in $V$-band, a calibration of reference stars in the field should allow the absolute flux to be calibrated on the image to a few per cent. This has not been possible on a full survey basis for SSDR1 since it would require re-calibrating several hundred EFOSC2 fields with reliable photometric measurements, or all sky reference catalogues. In the future of allsky digital surveys such as Pan-STARRS1 and SkyMapper, the existence of reference stars down to around $20^{m}$ will provide this improvement quite easily. We envisage future releases will improve on this.

The SSDR1 EFOSC2 and SOFI spectra and the SOFI images are available through the ESO archive server as formal ESO Phase 3 data. Instructions for accessing these data are available on the PESSTO website www.pessto.org. The reduced and calibrated EFOSC2 images are available from the PESSTO website, but not yet through the ESO archive. All 1D spectra will also available in the Weizmann Interactive Supernova data REPository (WISeREP Yaron \& Gal-Yam 2012).

Acknowledgements. This work is based on observations collected at the European Organisation for Astronomical Research in the Southern Hemisphere, Chile as part of PESSTO, (the Public ESO Spectroscopic Survey for Transient 


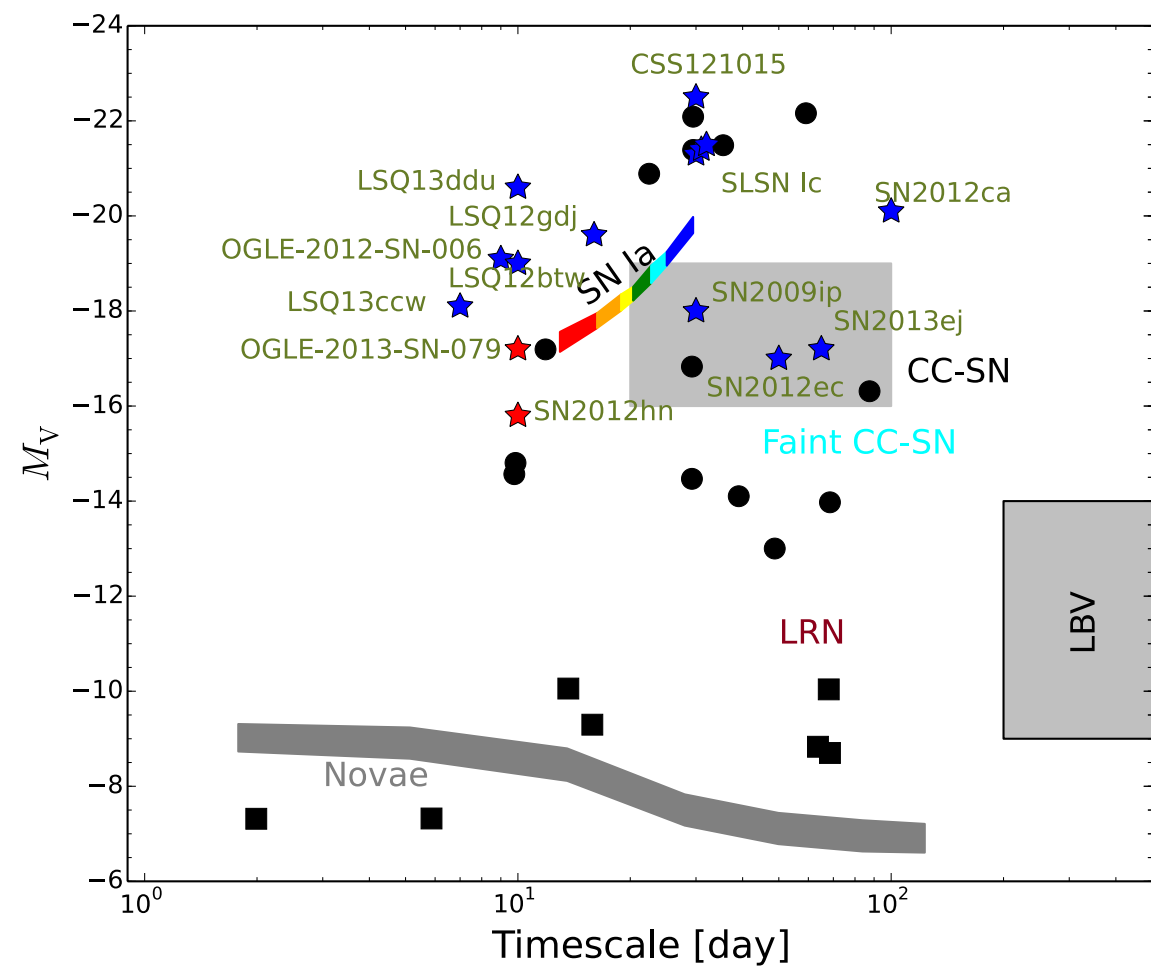

Fig. 21. PESSTO objects (filled blue stars) in the phase space of cosmic transients as originally developed by Kulkarni et al. (2007). The grey areas illustrate the known phase space for novae, luminous blue variable eruptions and core-collapse supernovae (the common types II and Ibc). The black squares show eruptive transients lying outside the nova regime, and the black dots are supernovae (normal and superluminous) or extreme erupitve events such as SN2008S (taken from Kulkarni \& Kasliwal 2009) The colour for each event represents the colour at peak brightness $(B-V$ or $g-r<$ 0.2 in blue; $B-V$ or $g-r>0.7$ in red).
Objects Survey) ESO programme 188.D-3003, 191.D-0935. Research leading to these results has received funding from the European Research Council under the European Union's Seventh Framework Programme (FP7/2007-2013)/ERC Grant agreement No. [291222] (PI : S. J. Smartt) and STFC grants ST/I001123/1 and ST/L000709/1. This research has made use of the SIMBAD database, operated at CDS, Strasbourg, France. M.J.C. acknowledges funding from the Australian Research Council Centre of Excellence for All-sky Astrophysics (CAASTRO), through project number CE110001020 MS acknowledges support from the Royal Society and EU/FP7-ERC grant No. [615929]. MF acknowledges support by the European Union FP7 programme through ERC grant number 320360 . S.B., E.C., A.P., L.T. and M.T. are partially supported by the PRININAF 2011 with the project Transient Universe: from ESO Large to PESSTO. We acknowledge CONICYT-Chile grants, Basal-CATA PFB-06/2007 (FEB), FONDECYT 1141218 (FEB) and 3140534 (SS), PCCI 130074 (FEB, SS), ALMA-CONICYT 31100004 (FEB, CRC), "EMBIGGEN" Anillo ACT1101 (FEB), Project IC120009 "Millennium Institute of Astrophysics (MAS) of the Iniciativa Científica Milenio del Ministerio de Economía, Fomento y Turismo (FEB, SS, CRC). A.G.-Y. is supported by the EU/FP7-ERC grant No. [307260], The Quantum Universe' I-Core programme by the Israeli Committee for planning and budgeting and the ISF, the Weizmann-UK making connections programme, and the Kimmel award. NER acknowledges support from the European Union Seventh Framework Programme (FP7/2007-2013) under grant agreement No. 267251 "Astronomy Fellowships in Italy" (AstroFIt). Support for LG, SG and HK is provided by the Ministry of Economy, Development, and Tourisms Millennium Science Initiative through grant IC12009, awarded to The Millennium Institute of Astrophysics, MAS. L.G., S.G. and H.K. acknowledge support by CONICYT through FONDECYT grants 3130680 and 3140563 , 3140566

\section{Appendix A: SSDR1 FITS Keywords description}

This section contains details of some of the more useful PESSTO specific SSDR1 FITS keywords and their definitions for users. It should be read in conjunction with the ESO Phase 3 User documentation (Retzlaff et al. 2013). The flux and wavelength related keywords are typically applicable to both SOFI and EFOSC2 data while some (such as the cosmic ray rejection flag) are applicable to one or the other only (EFOSC2 in this case). Their use in the two instruments should be self-explanatory in the descriptions.

\section{A.1. Number of exposures}

All PESSTO EFOSC2 spectra are extracted from single epoch exposures, we do not provided merged or co-added spectra in cases where multiple spectra are taken for EFOSC2. This is left to the users to decide. Hence for all EFOSC2 spectra:

SINGLEXP $=\mathrm{T} /$ TRUE if resulting from single exposure

PESSTO SOFI spectra are always taken in an ABBA dither pattern as described in Sect 6.2.4, and hence:

SINGLEXP $=\mathrm{F} /$ TRUE if resulting from single exposure

All PESSTO spectra are taken at a single epoch, hence:

M_EPOCH $=$ F / TRUE if resulting from multiple epochs

\section{A.2. Cosmic ray rejection}

PESSTO uses the Laplacian cosmic ray rejection algorithm ${ }^{14}$ of van Dokkum (2001) for EFOSC2 data (no cleaning is necessary for the SOFI detector). If the Boolean value is set to $\mathrm{T}$ as below, then the rejection algorithm has been applied, otherwise it has not. Note that in the spectral frames, only the central 200 pixels around the object are cleaned (i.e. central pixel \pm 100 pixels). Full frame cosmic ray cleaning is generally turned on for acquisition and photometric imaging, and again this is flagged with the following keyword.

LACOSMIC $=\mathrm{T} /$ TRUE if Laplacian cosmic ray rejection has been applied

\section{A.3. Wavelength calibration}

The particular arc frame used for wavelength calibration is always recorded for information using the ARC keyword. The number of arc lines used in the fit is given by LAMNLIN, and the root

${ }^{14}$ http://www.astro.yale.edu/dokkum/lacosmic/ 
mean square of the residuals to the fit is listed as LAMRMS, formally calculated as

LAMRMS $=\frac{\sqrt{\sum_{i=1}^{N} R_{i}^{2}}}{N}$

where $R_{i}$ is the residual of the wavelength fit for the $i$ th arcline and $N$ is the number of arc lines (LAMNLIN). This assumes that errors are randomly distributed and without any systematic errors, which is true as far as we can tell for EFOSC2 and SOFI. Hence the statistical uncertainty in the wavelength solution at any point is approximately given by the value SPEC_ERR, where

$$
\text { SPEC_ERR }=\frac{\text { LAMRMS }}{\sqrt{\text { LAMNLIN }}} .
$$

As described in Sect. 3.2.4 the wavelength positions of the skylines in the science frame (or telluric lines for bright standard stars) are checked and a linear shift is applied. This is listed in the keyword SHIFT in Angstroms. The precision of this is limited to $1 \AA$ and hence we set the keyword SPEC_SYE (the systematic error in the spectral coordinate system) that is found during the observation and reduction process to $1 \AA$. After this systematic SHIFT is applied to correct the skylines to rest, we find no further systematic effects in EFOSC2 wavelength calibration.

After the wavelength solution is determined and the SHIFT applied, the following values were inserted as keywords

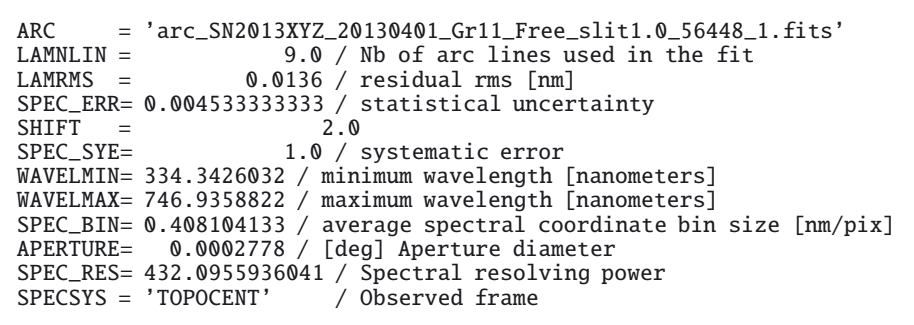

The dispersion is given by SPEC_BIN, determined simply from :

SPEC_BIN $=\frac{\text { WAVELMAX }- \text { WAVELMIN }}{n_{\text {pix }}}$

where $n_{\text {pix }}$ is the number of pixels in the array.

The slit width is given in degrees as the value APERTURE, and the resolving power is calculated from the nearest arc calibration frame (in time) to the science frame. We do not apply any velocity correction to the spectra, hence SPECSYS is set to topocentric.

\section{A.4. Detector characteristics}

As described in Sect. 3.1 and the read noise and gain have been remeasured for CCD\#40 on EFOSC2 and the correct values are written into the header as the following keywords.

DETRON =

GAIN

11.6 / Readout noise per output (e-)

EFFRON =

1.18 / Conversion from electrons to ADU

13.282436188 / Effective readout noise per output (e-)

GAIN is always the same in the EFOSC2 released data products, since they are single images and not combined. Similarly, the effective readnoise EFFRON is fairly constant since it only relies on the flats and biases used to detrend the data :

$\mathrm{EFFRON}=\operatorname{DETRON} \sqrt{1+\frac{1}{n_{\text {bias }}}+\frac{1}{n_{\text {flat }}}}$ where

$n_{\text {bias }}=$ number of bias frames making up the masterbias, $n_{\text {flat }}=$ number of flat-field frames making up the masterflat.

For SOFI imaging, the dithered images are median combined and hence the values for EFFRON and GAIN are calculated appropriately. In general, the images and spectra are shot noise limited from the high NIR background and readnoise is not a major factor.

\section{A.5. Instrument set-up and book keeping}

The object name is the primary name used by the supernova and transient community. Where it exists, an IAU name (e.g. SN 2013xy) is used, otherwise the survey specific names (e.g. LSQ12aaa), or the "potential" SN name from the CBAT "Transient Objects Confirmation Page" is employed. It is important to note that for all spectral frames the RA and Dec values refer to those of the target, not the telescope. However for all imaging frames the RA and Dec refer to the telescope pointing position.

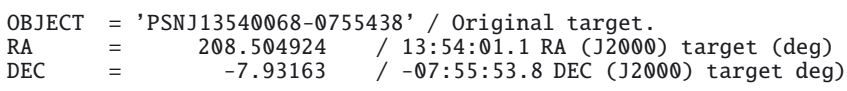

The ESO OB that created the science frames is recorded as OBID1. Since PESSTO provides the single epoch, individual spectra, there will always be only one OBID in the header. The title of the dataset is given as the MJD (of the observations), object name, grism, filter and slit combinations. In addition, the grism, filter and slit combinations are listed as below.

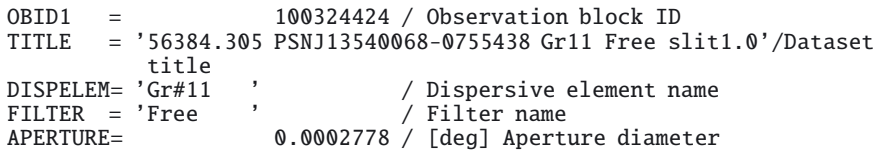

The relevant time stamps are listed below and are selfexplanatory, and are as defined in the ESO Science Data Products Standard (Retzlaff et al. 2013). We add our own AIRMASS keyword which is the mean airmass calculated at the midpoint of the exposure. This value is the one used in calculations of the sensitivity function, to flux calibrate the science spectra and to compute the zeropoints for EFOSC2 imaging.

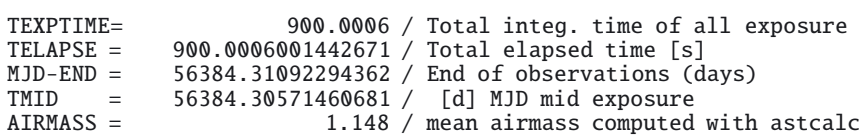

The version of the PESSTO pipeline which was used to reduce the data is recorded using the PROCSOFT keyword. The source code, installation guide, users manual and tutorial videos are available on the PESSTO wiki ${ }^{15}$. As discussed in Sect. 2.1, PESSTO immediately releases reduced data for all classification targets via WISeREP (Yaron \& Gal-Yam 2012) within $24 \mathrm{~h}$ of being taken. We label these "Fast" reductions, while the full reductions for SSDR1 are given an internal label of "Final" to distinguish them. This is recorded in the header keyword QUALITY. This publication is recorded as the primary scientific publication describing the data content.

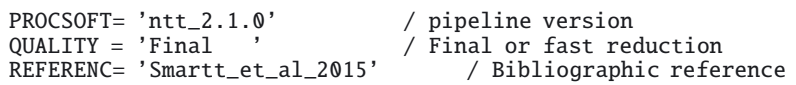

15 http://wiki.pessto.org/pessto-operation-groups/ data-reduction-and-quality-control-team 


\section{A.6. Flux calibration}

All objects extracted are by definition point sources hence the extended object keyword is always set to false. The spectra are flux calibrated and never normalised hence CONTNORM is always set to false and the FLUXCAL is set to ABSOLUTE. As described in the ESO Science Data Products Standard (Retzlaff et al. 2013) FLUXCAL should only be either ABSOLUTE or UNCALIBRATED. As PESSTO does not do wide slit observations to ensure that all flux is captured within the slit, we set TOT_FLUX to false always. The units of the flux calibration are in $\operatorname{erg~cm}{ }^{-2} \mathrm{~s}^{-1} \AA^{-1}$ in the FITS binary table spectra. The value for FLUXERR is set to either $15.2 \%$ for EFOSC2 or $34.7 \%$ for SOFI as described in Sects. 3.3 and 6.4.

The average signal-noise-ratio $(\mathrm{S} / \mathrm{N})$ per pixel is calculated by determining the $\mathrm{S} / \mathrm{N}$ in $N$ regions taken at $50 \AA$ intervals across the spectra and taking the mean. The number of regions $N$ is determined simply by (WAVELMAX-WAVELMIN)/50.

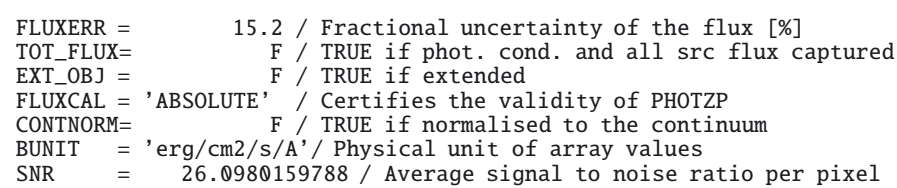

The associated 2D spectroscopic frame labelled as ASSON 1 and is submitted as an ancillary data product. This file is flux calibrated, and wavelength calibrated, and the units for that are in $10^{-20} \mathrm{erg} \mathrm{cm}^{-2} \mathrm{~s}^{-1} \AA^{-1}$

ASSON1 = 'SN2009ip_20130419 Gr11_Free_slit1.0_56448_1_si.fits'

ASSOC1 = 'ANCILLARY.2DSPECTRUM' / Category of associated file

\section{A.7. Imaging - photometric calibration}

The keywords PHOTZP, PHOTZPER, FLUXCAL, PHOTSYS are described above in Sect.4.1. Four other keywords are used to quantify the data.

\begin{tabular}{|c|c|c|}
\hline \multicolumn{3}{|l|}{ PHOTZP } \\
\hline PHOTZP = & 999 & error in PHOTZP \\
\hline FLUXCAL $=$ & 'ABSOLUTE' & Certifies the validity of PHOTZP \\
\hline PHOTSYS $=$ & 'VEGA & Photometric system VEGA or AB \\
\hline PSF_FWHM= & 1.32371928 & Spatial resolution (arcsec) \\
\hline ELLIPTIC $=$ & 0.131 & Average ellipticity of point sources \\
\hline ABMAGSAT $=$ & 0369 & Saturation limit point sources (AB mags) \\
\hline ARMACI TM- & 100 & 5-sigma limiting $A B$ magnitude point sour \\
\hline
\end{tabular}

The values for PSF_FWHM and ELLIPTIC are determined through a SEXTRACTOR (Bertin \& Arnouts 1996) measurement on the field which is automatically called within the PESSTO pipeline. The $5 \sigma$ limiting magnitude for a point source ABMAGLIM is derived from :

$$
\begin{aligned}
\text { MAGLIM } & =\text { PHOTZP }-2.5 \log \left(\frac{5}{(\text { GAIN })(\text { EXPTIME })^{2}}\right. \\
& \left.\times\left(N_{\text {pix }}\right)(\text { MBKG } \times \text { GAIN })+\left(\text { EFFRON }^{2} \times N_{\text {pix }}\right)^{1 / 2}\right)
\end{aligned}
$$

where $N_{\text {pix }}$ is the number of pixels in an aperture $\left(N_{\text {pix }}=\right.$ $\pi\left(\mathrm{PSF} \_F W H M / 0.24\right)^{2}$ for EFOSC2 where the 0.24 scaling factor is the pixel size in arcseconds; for SOFI this factor is 0.29 ) and MBKG is the median background in ADU estimated by SEXTRACTOR. We ignore extinction as a second-order effect in this calculation. In a small number of cases (around 3\% of the 2400 EFOSC2 images images) the images have short exposure times and low background such that after bias subtraction, the value of MBKG is negative. This may be due to bias drift as seen in Fig. 4, or a low enough background that read noise dominates and the overall value is below zero. In these cases Eq. (A.5) is still valid as the read noise will dominate.

The magnitude of a point source that will saturate at peak counts is given by the following equation. This assumes that saturation occurs at $60000 \mathrm{ADU}$ and that the volume under a 2D Gaussian is $2 \pi I_{0} \sigma^{2}$ (where $I_{0}$ is the peak intensity) and that $\mathrm{FWHM}=2 \sqrt{2 \ln 2} \sigma$, then

$$
\begin{aligned}
& \text { MAGSAT }=\text { PHOTZP }- 2.5 \log \left(\frac{\pi}{4 \ln 2}(60000\right. \\
&\left.- \text { MBKG })(\text { PSF_FWHM } / 0.24)^{2}\right),
\end{aligned}
$$

The saturation value of 60000 is assumed for EFOSC2 and for SOFI we assume 32000 ADU (from the SOFI manual; Lidman, et al. 2012) The MBKG value is simply the median background sky in ADU (the bias level has a negligible effect since it is $0.3 \%$ of the ADU 16-bit saturation level) and is recorded in the headers as such. The short exposure time problem, where MBKG may go negative, is not significant for this calculation. Again, the scaling factor of 0.24 is simply the pixel size for EFOSC2 and for SOFI it is 0.29 .

Although the header keywords are always listed as ABMAGSAT and ABMAGLIM, they should be interpreted in the photometric system given by PHOTSYS and not always assumed to be in the AB system.

\section{A.8. Imaging - astrometric calibration}

As described in Sect. 4.1, the keyword ASTROMET provides the number of catalogued stars used for the astrometric calibration and the rms of $\alpha$ and $\delta$ in arcseconds. The other keywords are mandatory ESO Science Data Products Standard keywords (Retzlaff et al. 2013). CSYER1 and CSYER2 should specify the contribution to the uncertainty of the astrometric calibration due to systematic errors intrinsic to the registration process. In our case this is dominated by the uncertainty intrinsic to the astrometric reference catalogues used. For data registered to the 2MASS point source catalogue we list the uncertainty in each axis as 100 milli-arcsec (Skrutskie et al. 2006) or 2.78E-05 degrees and and for USNO B1 it is 200 milli-arcsec (Monet et al. 2003) or 5.55E-05 degrees.

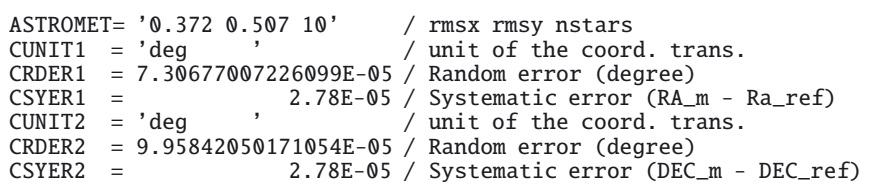

\section{Appendix B: Photometric nights}

The PESSTO observers record the night conditions in a night report which is publicly available on the PESSTO web pages (via the PESSTO wiki. The following table summarises that information. Where the conditions are labelled with "photometric", then the night was considered photometric in that there were no visible clouds at dusk or dawn and no obvious signs of clouds or transparency problems during the night. A "non-photometric" label means that the night was definitely not photometric, and a "?" means that there were no obvious transparency issues but with the information available we cannot be completely certain that it was photometric. 
Table B.1. Records on photometric nights.

\begin{tabular}{|c|c|c|c|}
\hline Night & "La Silla conditions & "Night & La Silla conditions \\
\hline 2013 Apr. 19 & photometric & 2012 Dec. 11 & photometric \\
\hline 2013 Apr. 18 & photometric & 2012 Dec. 06 & ? \\
\hline 2013 Apr. 17 & photometric & 2012 Dec. 05 & ? \\
\hline 2013 Apr. 13 & photometric & 2012 Dec. 04 & ? \\
\hline 2013 Apr. 12 & non-photometric & 2012 Dec. 03 & ? \\
\hline 2013 Apr. 11 & photometric & 2012 Nov. 22 & ? \\
\hline 2013 Apr. 05 & photometric & 2012 Nov. 21 & ? \\
\hline 2013 Apr. 04 & photometric & 2012 Nov. 20 & ? \\
\hline 2013 Apr. 03 & photometric & 2012 Nov. 14 & ? \\
\hline 2013 Apr. 02 & non-photometric & 2012 Nov. 13 & ? \\
\hline 2013 Apr. 01 & non-photometric & 2012 Nov. 12 & non-photometric \\
\hline 2013 Mar. 18 & non-photometric & 2012 Nov. 07 & non-photometric \\
\hline 2013 Mar. 17 & ? & 2012 Nov. 06 & photometric \\
\hline 2013 Mar. 16 & photometric & 2012 Nov. 05 & photometric \\
\hline 2013 Mar. 12 & photometric & 2012 Nov. 04 & photometric \\
\hline 2013 Mar. 11 & photometric & 2012 Oct. 22 & ? \\
\hline 2013 Mar. 10 & photometric & 2012 Oct. 21 & non-photor \\
\hline 2013 Mar. 05 & photo & 2012 Oct. 20 & $?$ \\
\hline 2013 Mar. 04 & photometric & 2012 Oct. 16 & non-photometric \\
\hline 2013 Mar. 03 & tometric & 2012 Oct. 15 & $?$ \\
\hline 2013 Mar. 02 & otometric & 2012 Oct. 14 & ? \\
\hline 2013 Mar. 01 & non-photometric & 2012 Oct. 09 & non-photometric \\
\hline 2013 Feb. 21 & photo & 2012 Oct. 08 & ? \\
\hline 2013 Feb. 20 & photometric & 2012 Oct. 07 & non-photometric \\
\hline 2013 Feb. 19 & non-photometric & 2012 Oct. 06 & non-photometric \\
\hline 2013 Feb. 08 & non-photometric & 2012 Sep. 25 & non-photometric \\
\hline 2013 Feb. 07 & non-photometric & 2012 Sep. 24 & photometric \\
\hline 2013 Feb. 06 & non-photometric & 2012 Sep. 23 & non-photometric \\
\hline 2013 Jan. 30 & non-photometric & 2012 Sep. 22 & non-photometric \\
\hline 2013 Jan. 29 & ? & 2012 Sep. 17 & non-photometric \\
\hline 2013 Jan. 28 & ? & 2012 Sep. 16 & non-photometric \\
\hline 2013 Jan. 27 & $?$ & 2012 Sep. 15 & non-photometric \\
\hline 2013 Jan. 21 & non- 1 & 2012 Sep. 09 & phot \\
\hline 2013 Jan. 20 & non-photometric & 2012 Sep. 08 & photometric \\
\hline 2013 Jan. 19 & non-photometric & 2012 Sep. 07 & non-photometric \\
\hline 2013 Jan. 13 & non-photometric & 2012 Sep. 06 & non-photometric \\
\hline 2013 Jan. 12 & ? & 2012 Aug. 26 & non-photometric \\
\hline 2013 Jan. 11 & ? & 2012 Aug. 25 & non-photometric \\
\hline 2013 Jan. 04 & ? & 2012 Aug. 24 & photometric \\
\hline 2013 Jan. 03 & ? & 2012 Aug. 18 & photometric \\
\hline 2013 Jan. 02 & ? & 2012 Aug. 17 & non-photometric \\
\hline 2013 Jan. 01 & ? & 2012 Aug. 16 & non-photometric \\
\hline 2012 Dec. 22 & ? & 2012 Aug. 10 & photometric \\
\hline 2012 Dec. 21 & . & 2012 Aug. 09 & non-photometric \\
\hline 2012 Dec. 20 & ? & 2012 Aug. 08 & non-photometric \\
\hline 2012 Dec. 13 & & 2012 Aug. 07 & photometric \\
\hline & & & \\
\hline
\end{tabular}

\section{Appendix C: Software for reading FITS binary tables}

As described in Sects. 5.2 and 6.6, the PESSTO spectra from the ESO Science Archive Facility are in FITS binary table format. Not all astronomical software routines can read this format easily. Listed here are some examples of software that can be used. This information is linked from the PESSTO survey home page and there are links there to follow to get the relevant software.

1. The new IRAF external package SPTABLE is able to read, display, and analyse (via the onedspec and rv packages).

2. Fv is a graphical program for viewing and editing any FITS format image or table available from NASA's High Energy Astrophysics Science Archive Research Center (HEASARC).
3. VOSpec is a multi-wavelength spectral analysis tool from the ESA Virtual Observatory team.

4. SPLAT-VO is a Virtual Observatory enabled package that originated in STARLINK and is now released as part of the German Astrophysical Virtual Observatory (GAVO)

5. IDL and python can also read FITS binary tables through the IDL Astronomy User's Library at Goddard and through pyfits respectively.

6. Further details on all the above are linked from the PESSTO website $^{16}$.

\section{References}

Ahn, C. P., Alexandroff, R., Allende Prieto, C., et al. 2012, ApJS, 203, 21 Baltay, C., Rabinowitz, D., Hadjiyska, E., et al. 2012, The Messenger, 150, 34 Baltay, C., Rabinowitz, D., Hadjiyska, E., et al. 2013, PASP, 125, 683 Benetti, S., Nicholl, M., Cappellaro, E., et al. 2014, MNRAS, 441, 289 Berger, E., Chornock, R., Lunnan, R., et al. 2012, ApJ, 755, L29

Bertin, E., \& Arnouts, S. 1996, A\&AS, 117, 393

Bertin, E., Mellier, Y., Radovich, M., et al. 2002, in Astronomical Data Analysis Software and Systems XI, ed. D. A. Bohlender, D. Durand, \& T. H. Handley, ASP Conf. Ser., 281, 228

Blondin, S., \& Tonry, J. L. 2007, ApJ, 666, 1024

Boroson, T., Brown, T., Hjelstrom, A., et al. 2014, in SPIE Conf. Ser., 9149

Botticella, M. T., Trundle, C., Pastorello, A., et al. 2010, ApJ, 717, L52

Cao, Y., Kasliwal, M. M., Arcavi, I., et al. 2013, ApJ, 775, L7

Childress, M. J., Scalzo, R. A., Sim, S. A., et al. 2013, ApJ, 770, 29

Chomiuk, L., Chornock, R., Soderberg, A. M., et al. 2011, ApJ, 743, 114

Clough, S. A., Shephard, M. W., Mlawer, E. J., et al. 2005, J. Quant. Spectr. Rad. Transf., 91, 233

DePoy, D. L., Atwood, B., Belville, S. R., et al. 2003, in Instrument Design and Performance for Optical/Infrared Ground-based Telescopes, eds. M. Iye, \& A. F. M. Moorwood, SPIE Conf. Ser., 4841, 827

Drake, A. J., Djorgovski, S. G., Mahabal, A., et al. 2009, ApJ, 696, 870

Drake, A. J., Djorgovski, S. G., Prieto, J. L., et al. 2010, ApJ, 718, L127

Drake, A. J., Djorgovski, S. G., Mahabal, A., et al. 2011, ApJ, 735, 106

Ellis, R. S., Sullivan, M., Nugent, P. E., et al. 2008, ApJ, 674, 51

Fraser, M., Inserra, C., Jerkstrand, A., et al. 2013, MNRAS, 433, 1312

Gal-Yam, A., Kasliwal, M. M., Arcavi, I., et al. 2011, ApJ, 736, 159

Gal-Yam, A., Arcavi, I., Ofek, E. O., et al. 2014, Nature, 509, 471

Gezari, S., Chornock, R., Rest, A., et al. 2012, Nature, 485, 217

Harutyunyan, A. H., Pfahler, P., Pastorello, A., et al. 2008, A\&A, 488, 383

Hodgkin, S. T., Wyrzykowski, L., Blagorodnova, N., \& Koposov, S. 2013, Roy. Soc. Lond. Philosoph. Trans. Ser. A, 371, 20239

Howell, D. A., Sullivan, M., Perrett, K., et al. 2005, ApJ, 634, 1190

Inserra, C., Smartt, S. J., Jerkstrand, A., et al. 2013, ApJ, 770, 128

Inserra, C., Smartt, S. J., Scalzo, R., et al. 2014, MNRAS, 437, L51

Jester, S., Schneider, D. P., Richards, G. T., et al. 2005, AJ, 130, 873

Kaiser, N., Burgett, W., Chambers, K., et al. 2010, in SPIE Conf. Ser., 7733

Kasliwal, M. M., Kulkarni, S. R., Gal-Yam, A., et al. 2010, ApJ, 723, L98

Keller, S. C., Schmidt, B. P., Bessell, M. S., et al. 2007, PASA, 24, 1

Klotz, A., Vachier, F., \& Boër, M. 2008, Astron. Nach., 329, 275

Kozłowski, S., Udalski, A., Wyrzykowski, Ł., et al. 2013, Acta Astron., 63, 1

Kulkarni, S., \& Kasliwal, M. M. 2009, in Astrophysics with All-Sky X-Ray Observations, eds. N. Kawai, T. Mihara, M. Kohama, \& M. Suzuki, 312 Kulkarni, S. R., Ofek, E. O., Rau, A., et al. 2007, Nature, 447, 458

Law, N. M., Kulkarni, S. R., Dekany, R. G., et al. 2009, PASP, 121, 1395

Leaman, J., Li, W., Chornock, R., \& Filippenko, A. V. 2011, MNRAS, 412, 1419

Li, W., Leaman, J., Chornock, R., et al. 2011, MNRAS, 412, 1441

Lidman, C., Cuby, J.-G., Vanzi, L., et al. 2012, LSO-MAN-ESO-40100-0004, 2.3

Lipunov, V., Kornilov, V., Gorbovskoy, E., et al. 2010, Adv. Astron., 349171

Lord, S. 1992, NASA Technical Memorandum, 103957

Magnier, E. A., Schlafly, E., Finkbeiner, D., et al. 2013, ApJS, 205, 20

Maguire, K., Sullivan, M., Patat, F., et al. 2013, MNRAS, 436, 222

Maguire, K., Sullivan, M., Pan, Y.-C., et al. 2014, MNRAS, 444, 3258

Maund, J. R., Fraser, M., Smartt, S. J., et al. 2013, MNRAS, 431, L102

Monaco, L., Snodgrass, C., Schmidtobreick, L., \& Saviane, I. 2012, LSO-MANESO-36100-0004, 3.6

Monet, D. G., Levine, S. E., Canzian, B., et al. 2003, AJ, 125, 984

Moorwood, A., Cuby, J.-G., \& Lidman, C. 1998, The Messenger, 91, 9

Nicholl, M., Smartt, S. J., Jerkstrand, A., et al. 2014, MNRAS, 444, 2096

Nugent, P. E., Sullivan, M., Cenko, S. B., et al. 2011, Nature, 480, 344

\footnotetext{
16 http://archive. eso.org/cms/eso-data/help/1dspectra. html
} 
O’Brien, P. T., \& Smartt, S. J. 2013, Roy. Soc. Lond. Philosoph. Trans. Ser. A, 371, 20498

Ofek, E. O., Sullivan, M., Cenko, S. B., et al. 2013, Nature, 494, 65

Pastorello, A., Smartt, S. J., Mattila, S., et al. 2007, Nature, 447, 829

Pastorello, A., Smartt, S. J., Botticella, M. T., et al. 2010, ApJ, 724, L16

Pastorello, A., Cappellaro, E., Inserra, C., et al. 2013, ApJ, 767, 1

Patat, F., Moehler, S., O’Brien, K., et al. 2011, A\&A, 527, A91

Perez, F., Bagish, A., Bredthauer, G., et al. 2012, in SPIE Conf. Ser., 8444

Pignata, G., Maza, J. Hamuy, M., et al. 2009, AIP Conf. Proc., 1111, 551

Rau, A., Kulkarni, S. R., Law, N. M., et al. 2009, PASP, 121, 1334

Reichart, D., Nysewander, M., Moran, J., et al. 2005, Nuovo Cimento C Geophysics Space Physics C, 28, 767

Retzlaff, J., Delmotte, N., Arnaboldi, M., \& Romaniello, M. 2013, GEN-SPEESO-33000-5335, 5

Scalzo, R. A., Childress, M., Tucker, B., et al. 2014, MNRAS, 445, 30

Shappee, B. J., Prieto, J. L., Grupe, D., et al. 2014, ApJ, 788, 48

Skrutskie, M. F., Cutri, R. M., \& Stiening, R. 2006, AJ, 131, 1163

Smartt, S. J. 2009, ARAA, 47, 63

Smartt, S. J., Smith, K. W., Wright, D., et al. 2014, The Astronomer's Telegram, 5850,1

Soderberg, A. M., Berger, E., Page, K. L., et al. 2008, Nature, 453, 469

Steele, I. A., Smith, R. J., Rees, P. C., et al. 2004, in Ground-based Telescopes, ed. J. M. Oschmann, Jr., SPIE Conf. Ser., 5489, 679

Stritzinger, M., Suntzeff, N. B., Hamuy, M., et al. 2005, PASP, 117, 810

Sullivan, M. 2013, Astron. Geophys., 54, 060006

Tomasella , L., Benetti, S., Cappellaro, E., et al. 2014, Astron. Nachr., 335, 841

Valenti, S., Pastorello, A., Cappellaro, E., et al. 2009, Nature, 459, 674

Valenti, S., Sand, D., Pastorello, A., et al. 2014a, MNRAS, 438, L101

Valenti, S., Yuan, F., Taubenberger, S., et al. 2014b, MNRAS, 437, 1519

van Dokkum, P. G. 2001, PASP, 113, 1420

Wyrzykowski, Ł., Kostrzewa-Rutkowska, Z., Kozłowski, S., et al. 2014, Acta Astron., 64, 197

Yaron, O., \& Gal-Yam, A. 2012, PASP, 124, 668

1 Astrophysics Research Centre, School of Mathematics and Physics, Queen's University Belfast, Belfast BT7 1NN, UK

e-mail: s.smartt@qub.ac.uk

2 Las Cumbres Observatory Global Telescope Network, 6740 Cortona Dr., Suite 102, Goleta, California 93117, USA

3 Department of Physics, University of California Santa Barbara, Santa Barbara, CA 93106, USA

4 Institute of Astronomy, University of Cambridge, Madingley Road, Cambridge CB3 0HA, UK

5 School of Physics and Astronomy, University of Southampton, Southampton SO17 1BJ, UK

6 INAF-Osservatorio Astronomico di Padova, Vicolo del l'Osservatorio 5, 35122 Padova, Italy

7 Benoziyo Center for Astrophysics, Weizmann Institute of Science, 76100 Rehovot, Israel

8 INAF-Osservatorio Astronomico di Trieste, via G.B. Tiepolo 11, 34143 Trieste, Italy

9 Max-Planck-Institut fur Astrophysik, Karl-Schwarzschildstr. 1, 85748 Garching, Germany

10 European Southern Observatory, Alonso de Cordova 3107, Vitacura, Santiago

11 Laboratoire de Physique Nucléaire et des Hautes Énergies, Université Pierre et Marie Curie Paris 6, Université Paris Diderot Paris 7, CNRS-IN2P3, 4 place Jussieu, 75252 Paris Cedex 05, France

12 Physics Department, Yale University, New Haven, CT 06520, USA

13 INAF-Osservatorio astronomico di Capodimonte, V. Moiariello 16, 80131 Napoli, Italy

14 Dip. di Fisica and ICRA, Sapienza Universit ă di Roma, Piazzale Aldo Moro 5, 00185 Rome, Italy
15 Instituto de Astrofísica, Facultad de Física, Pontificia Universidad Católica de Chile, 306, Santiago 22, Chile

16 Millennium Institute of Astrophysics, Vicuña Mackenna 4860, 7820436 Macul, Santiago, Chile

17 Space Science Institute, 4750 Walnut Street, Suite 205, Boulder, Colorado 80301, USA

18 Astrophysics Research Institute, Liverpool John Moores University, Liverpool L3 5RF, UK

19 Departamento de Ciencias Fisicas, Universidad Andres Bello. Avda. Republica 252, Santiago, Chile

20 Research School of Astronomy and Astrophysics, Australian National University, Canberra, ACT 2611, Australia

21 Institut d'Astrophysique de Paris, CNRS, and Université Pierre et Marie Curie, 98bis boulevard Arago, 75014 Paris, France

22 Institut de Ciencies de l'Espai (IEEC-CSIC), Facultat de Cincies, Campus UAB, 08193 Bellaterra, Spain

23 Departamento de Astronomía - Universidad de Chile, Camino el Observatorio 1515, Santiago, Chile

24 Finnish Centre for Astronomy with ESO (FINCA), University of Turku, Vaisalantie 20, 21500 Piikkio, Finland

25 Dark Cosmology Centre, Niels Bohr Institute, University of Copenhagen, Juliane Maries vej 30, 2100 Copenhagen, Denmark

26 Department of Astronomy and the Oskar Klein Centre, Stockholm University, AlbaNova, 10691 Stockholm, Sweden

27 University of Oxford Astrophysics, Denys Wilkinson Building, Keble Road, Oxford OX1 3RH, UK

28 INAF Astronomical Observatory of Rome, via Frascati 33, 00040 Monte Porzio Catone (RM), Italy

29 European Southern Observatory, KarlSchwarzschildStrasse 2, 85748 Garching, Germany

30 Gemini Observatory, Southern Operations Center, Casilla 603, La Serena, Chile

31 Lawrence Berkeley National Laboratory, Berkeley, California 94720, USA

32 ARC Centre of Excellence for All-sky Astrophysics (CAASTRO), Australia

33 Institut für Theoretische Physik und Astrophysik, Universität Würzburg, Emil-Fischer-Str. 31, 97074 Würzburg, Germany

34 Institut für Mathematik, Universität Würzburg, Emil-FischerStr. 30, 97074 Würzburg, Germany

35 Warsaw University Observatory, Al. Ujazdowskie 4, 00-478 Warszawa, Poland

36 Tuorla Observatory, Department of Physics and Astronomy, University of Turku, Väiäläntie 20, 21500 Piikkiö, Finland

37 Millennium Institute of Astrophysics, Universidad de Chile, Casilla 36-D, Santiago, Chile

38 Università degli Studi di Padova, Dipartimento di Fisica e Astronomia, Vicolo dellOsservatorio 2, 35122 Padova, Italy

39 Institut für Physik, Humboldt-Universität zu Berlin, Newtonstr. 15, 12489 Berlin, Germany

40 Physikalisches Institut, Universität Bonn, Nußallee 12, 53115 Bonn, Germany

41 Department of Astronomy, University of California, Berkeley, CA 94720, USA

42 Departamento de Investigación Básica, CIEMAT, Av da. Complutense 40, 28040 Madrid, Spain

43 Carnegie Observatories, Las Campanas Observatory, Colina El Pino, Casilla 601, Chile

44 Department of Physics and Astronomy, Aarhus University, Ny Munkegade, 8000 Aarhus C, Denmark

45 Department of Physics, University of Warwick, Coventry CV4 7AL, UK 